\title{
Special Lagrangians, stable bundles and mean curvature flow
}

\author{
R. P. Thomas and S.-T. YAU
}

\begin{abstract}
We make a conjecture about mean curvature flow of Lagrangian submanifolds of Calabi-Yau manifolds, expanding on that of [Th]. We give new results about the stability condition in [Th], and propose a Jordan-Hölder-type decomposition of (special) Lagrangians. The main results are the uniqueness of special Lagrangians in hamiltonian deformation classes of Lagrangians, under mild conditions, and a proof of the conjecture in some cases with symmetry: mean curvature flow converging to Shapere-Vafa's examples of SLags.
\end{abstract}

\section{Introduction.}

Fix a Calabi-Yau manifold $X$ with a holomorphic $(n, 0)$-form $\Omega$. In [Th] a stability condition for Lagrangians in $X$ was described, conjectured to be equivalent to the existence of a special Lagrangian (SLag) in the hamiltonian deformation class of a fixed Lagrangian. This was motivated by an infinite dimensional set-up in which $U(1)$ gauge transformations act on the (infinite dimensional) space of Lagrangians with flat $U(1)$ connections on them. There is a natural complex structure and symplectic form on this space and, ignoring issues of integrability of these structures (see [Th]), the formal complexification of the $U(1)$ gauge transformations gives hamiltonian deformations of the Lagrangian, with moment map the $n$-form $\left.\operatorname{Im} \Omega\right|_{L}$. The stability condition was motivated by an example of Joyce and the 'angle criterion', in terms of splittings of the Lagrangian into Seidel's graded Lagrangian connect sums (as defined in Section 3 below) and family versions thereof, with a certain phase inequality. This led to a conjecture, a sort of globalised version of the angle criterion $[\mathrm{L}],[\mathrm{N}]$, that the hamiltonian deformation class of a Lagrangian should contain a SLag if and only if the Lagrangian is stable; this SLag representative should then be unique. Here we expand on the conjecture and relate it to mean curvature flow. It was verified for the simplest case $T^{2}$ in [Th]; here we prove it in a series of $n$ dimensional examples with symmetry (Theorem 7.6), and prove uniqueness 
of smooth SLags in hamiltonian deformation classes whose Floer cohomology [FO3] is defined (Theorem 4.3).

We write $\approx$ for "in the same hamiltonian deformation class as", and use $\sim$ for the isomorphism $T^{*} L \rightarrow T L$ induced by the metric on a Riemannian manifold $L$. Restricting the Ricci-flat metric on a Calabi-Yau manifold $(X, \Omega)$ to a Lagrangian submanifold $L$ we get an induced volume form vol on $L$, and by a short calculation

$$
\left.\Omega\right|_{L}=e^{i \theta} \mathrm{vol}
$$

defines an $S^{1}$-valued function $\theta$ on $L$, the phase function of $L$. A grading of $L$ is a lift of $\theta$ to a real valued function. By Lagrangian we will always mean graded Lagrangian (thus the Maslov class of the Lagrangian, which is the class of $d \theta$ in $H^{1}(L ; 2 \pi \mathbb{Z})$, is assumed to vanish, and we have chosen a lift of $\theta$ ). $L$ is special Lagrangian (SLag) if $\theta$ is a constant; equivalently, replacing $\Omega$ by $e^{-i \theta} \Omega,\left.\operatorname{Im} \Omega\right|_{L} \equiv 0$. An average, cohomological, measure of the phase of a homology class $[L]$ is given by taking the phase of the complex number $\int_{L} \Omega$; for $L$ graded with the variation of $\theta$ less than $2 \pi$, this lifts naturally to give a real number $\phi(L)$, which is the phase of any SLag in the same homology class.

We should point out that as in [Th], we do not fully understand the role of holomorphic discs in the theory. These are of course crucial in the definition and hamiltonian deformation invariance of Floer cohomology; until this is fully set up [FO3] and all of its expected properties (such as the spectral sequences of $[\mathrm{Oh} 2]$ and $[\mathrm{P}]$ ) are proved and extended to the Calabi-Yau case, some of the arguments below are necessarily conjectural; it will be clear which ones. We also deal exclusively with smooth (S)Lags; how to modify our constructions to include singularities is an important question. Using only (family) Lagrangian connect sums as the degenerations necessary to describe stability of Lagrangians is also probably too restrictive, studying other singularities and splittings may also be necessary; the conjecture in this paper is probably just the first step in understanding SLags in hamiltonian isotopy classes.

Acknowledgements. The symplectic ideas and suggestions of Paul Seidel have been absolutely invaluable throughout this work. We have also benefitted from comments from Kenji Fukaya, Edward Goldstein, Spiro Karigiannis, Conan Leung, Jun Li, Elizabeth Mann, Yong-Geun Oh and Xiao Wei Wang, and would like to thank Mike Gage for the reference [An]. The first author is supported by a Royal Society university research fellowship and by Imperial College, London; the second author is supported by DOE grant DE-FG02-88ER35065 and NSF grant DMS-9803347. 


\section{Mean curvature flow.}

We first give a well-known geometric calculation which we learnt from unpublished lectures of Rick Schoen on his work with Jon Wolfson, but which dates back at least as far as [HaL], [Oh1] and others.

Lemma 2.1. In the above notation, the mean curvature vector of the Lagrangian $L \subset X$ is $M C V=J \widetilde{d \theta}$.

Proof We want to show that for any vector $X$ tangent to $L, X \theta=$ $-\langle\mathrm{MCV}, J X\rangle$.

Picking an orthonormal basis of $T_{p} L$ and parallel transporting it along rays in $L$ to a frame field $\left(e_{i}\right),\left(e_{i}, J e_{i}\right)$ forms a local basis for $T X$ around $p$. Letting $\left(f_{j}, g_{j}=-f_{j} \circ J\right)$ be the dual basis of 1-forms, it is clear that at $p$,

$$
\Omega=e^{-i \theta} \bigwedge_{j}\left(f_{j}+i g_{j}\right)
$$

with $\theta$ the phase function of $L$. Since $\Omega$ is parallel, $\nabla_{X} \Omega=0$ yields

$$
\begin{aligned}
i X(\theta) \bigwedge_{j}\left(f_{j}+i g_{j}\right) & =\sum_{k}\left(f_{1}+i g_{1}\right) \wedge \ldots \wedge \nabla_{X}\left(f_{k}+i g_{k}\right) \wedge \ldots \wedge\left(f_{n}+i g_{n}\right) \\
& =\sum_{k}\left[\nabla_{X}\left(f_{k}+i g_{k}\right)\left(\frac{1}{2}\left(e_{k}-i J e_{k}\right)\right)\right] \bigwedge_{j}\left(f_{j}+i g_{j}\right) .
\end{aligned}
$$

Taking covariant derivatives on the Calabi-Yau (i.e. not on $L$ ), we have

$$
-\langle\mathrm{MCV}, J X\rangle=-\left\langle\sum_{i} \nabla_{e_{i}} e_{i}, J X\right\rangle=\sum_{i}\left\langle\nabla_{e_{i}} J e_{i}, X\right\rangle,
$$

since $J$ is both skew adjoint and parallel. But as $J e_{i}$ and $X$ are orthogonal, this is

$$
-\sum_{i}\left\langle J e_{i}, \nabla_{e_{i}} X\right\rangle=\sum_{i}\left\langle e_{i}, J \nabla_{X} e_{i}\right\rangle,
$$

as we may choose $X$ to have zero bracket with the $e_{i}$ s.

So comparing with (2.2) we are left with showing that

$$
\sum_{k}\left[\nabla_{X}\left(f_{k}+i g_{k}\right)\left(\frac{1}{2}\left(e_{k}-i J e_{k}\right)\right)\right]=i \sum_{i}\left\langle e_{i}, J \nabla_{X} e_{i}\right\rangle,
$$

i.e. that $\left(\nabla_{X}\left(f_{k}+i g_{k}\right)\right)\left(e_{k}-i J e_{k}\right)=2 i\left\langle e_{k}, J \nabla_{X} e_{k}\right\rangle$. 
But $\left(f_{k}+i g_{k}\right)\left(e_{k}-i J e_{k}\right)=2$ is a constant, so the left hand side is $-\left(f_{k}+i g_{k}\right)\left(\nabla_{X}\left(e_{k}-i J e_{k}\right)\right)=-f_{k}\left(\nabla_{X}\left(-i J e_{k}\right)\right)-i g_{k}\left(\nabla_{X} e_{k}\right)$; the other terms vanish as $\nabla_{X} e_{i}$ was chosen to be perpendicular to $L$. Using $\nabla_{X} J=0$ and recalling that $g_{k}=-f_{k} \circ J$, we obtain $2 i\left\langle e_{k}, J \nabla_{X} e_{k}\right\rangle$.

Another simple but important result is how the phase $\theta$ and volume form $\operatorname{vol}_{L}$ vary under a hamiltonian deformation $J \widetilde{d h}$ of $L$. Such calculations appear in various forms in [Oh1], [Sm], for instance; we give short geometric proofs for completeness.

Lemma 2.3. Under a hamiltonian deformation $J \widetilde{d h}$ of a Lagrangian $L$, we have

$$
\begin{aligned}
\frac{d}{d t} \theta & =-\Delta_{L}(h), \\
\frac{d}{d t} \operatorname{vol}_{L} & =-\langle d \theta, d h\rangle \operatorname{vol}_{L} .
\end{aligned}
$$

Proof Take real and imaginary parts of $e^{-i \theta}$ times the following:

$$
\begin{aligned}
i \dot{\theta} e^{i \theta} \operatorname{vol}_{L} & \left.+e^{i \theta} \frac{d}{d t} \operatorname{vol}_{L}=\frac{d}{d t}\left(e^{i \theta} \operatorname{vol}_{L}\right)=\left.\left(\mathcal{L}_{J \widetilde{d h}} \Omega\right)\right|_{L}=d(J \widetilde{d h}\lrcorner \Omega\right)\left.\right|_{L} \\
& \left.\left.=i d\left(e^{i \theta} \widetilde{d h}\right\lrcorner \operatorname{vol}_{L}\right)=-e^{i \theta} d \theta \wedge(\widetilde{d h}\lrcorner \operatorname{vol}_{L}\right)-i e^{i \theta} d^{*} d h \operatorname{vol}_{L} .
\end{aligned}
$$

Setting $\Delta_{L}=d^{*} d\left(=-\sum_{i} \partial_{x_{i}}^{2}\right.$ in geodesic coordinates $)$ gives the result.

We next show that, given a suitable metric on the Lie algebra $C^{\infty}(L, \mathbb{R}) / \mathbb{R}$, the gradient flow of the norm square $-|m|^{2}$ of the moment $\operatorname{map} m=\left.\operatorname{Im} \Omega\right|_{L}$ of $[\mathrm{Th}]$ is mean curvature flow. The following standard calculation, applicable in any Kähler reduction picture, shows that the gradient flow of $-|m|^{2}$ is given by $J X_{m^{*}}$, where $J$ is the complex structure, $m^{*}$ is the element of the Lie algebra $C^{\infty}(L)$ corresponding to the moment map $m=\left.\operatorname{Im} \Omega\right|_{L}$ in the dual of the Lie algebra under the metric on $C^{\infty}(L)$, and $X_{m^{*}}$ is its induced action on the space (Lagrangians with flat $U(1)$ connections on them).

$$
X\left(-|m|^{2}\right)=-X\left(m\left(m^{*}\right)\right)=-\omega\left(X, X_{m^{*}}\right)=\left\langle X, J X_{m^{*}}\right\rangle .
$$

By the definition of the group action in [Th], this deformation $J X_{m^{*}}$ is just the hamiltonian deformation of the Lagrangian $L$ with hamiltonian function $m^{*}$ on $L$. 
Choosing the volume form $\left.\operatorname{Re} \Omega\right|_{L}$ on $L$ to define an $L^{2}$ metric on $C^{\infty}(L)$ gives $m^{*}=\tan \theta$, since $m=\left.\operatorname{Im} \Omega\right|_{L}=\sin \theta \mathrm{vol}=\left.\tan \theta \operatorname{Re} \Omega\right|_{L}$. Similarly using the induced Riemannian volume form vol gives $m^{*}=\sin \theta$, while using

$$
\frac{\sin \theta}{\theta} \text { vol }
$$

as volume form on $L$ yields $m^{*}=\theta$. Any of these are suitable for small phase $\theta: L \rightarrow \mathbb{R}$, and give similar flows down which the moment map decreases. The last one, however, is precisely mean curvature flow, by Lemma 2.1.

This and the previous lemma show that under mean curvature flow, the phase $\theta$ satisfies a (time dependent) heat equation while the Riemannian volume form decreases (as usual):

$$
\begin{aligned}
\dot{\theta} & =-\Delta \theta, \\
\frac{d}{d t} \operatorname{vol}_{L} & =-|d \theta|^{2} \operatorname{vol}_{L} .
\end{aligned}
$$

We therefore obtain a maximum principle for $\theta$, whose range must always decrease, but it is important to note that the Laplacian $\Delta$ is time dependent as the metric on $L$ used to define it varies.

From these follow a series of identities and estimates, many of which we use later, but none are strong enough to give long term existence of the mean curvature flow, and with good reason. Mean curvature flow is a complicated and much-studied subject (understood only in codimension 1, dimension 1 [Gr], and, in special cases, in two dimensions [Wa]), with known examples of finite time blow-up. While we might expect it to behave better for Lagrangians (locally functions of one variable instead of $n$ ), examples in Section 6 show similar phenomena. But in our examples there will be a way round these problems, and we will be able to make a conjecture about the flow which may help in its study.

\section{Connect sums and Floer gradings.}

The stability definition in [Th] made extensive use of graded Lagrangian connect sums [S2]; a description of these and their relationship to Floer cohomology will be important again here, as will knowledge of the Floer index of Lagrangian intersections. We fix our conventions and definitions now; in some places these differ in orientation from some of the mirror symmetry literature and [S2]; the problem seems to be deciding on whether to use the standard symplectic form $d x d y$ on $T^{2}$, or the equally standard $d p d q=-d x d y$ considering it as the cotangent bundle of its SYZ base $S^{1}$ (divided by a lattice) [SYZ]. 


\subsection{The connect sum.}

Suppose we have two Lagrangians $L_{1}, L_{2}$ hamiltonian isotoped to intersect transversally in a finite number of points. We will work at one of these points $p$. There we can pick a local Darboux chart with coordinates $\left(x_{i}, y_{i}\right)$ and symplectic form $\sum_{i} d x_{i} \wedge d y_{i}$ such that $L_{2}=\left\{y_{i}=0\right\}$ is the $x$-axes, and

$$
L_{1}=\left\{y_{i}=\tan (\alpha) x_{i}\right\}
$$

for some $\alpha \in(0, \pi)$. (It would be more usual to use. $\alpha=\pi / 2$, of course, but that situation can be moved to this one by an obvious symplectic (shear) transformation).

Using $z_{i}=x_{i}+i y_{i}$ coordinates to set up the obvious isomorphism to $\mathbb{C}^{n}$ (notice that this complex structure and that inherited from $X$ may be different), the $L_{i}$ are

$$
\begin{aligned}
L & =e^{i \alpha}[0, \infty) \cdot S^{n-1} \\
& :=\left\{z_{j}=r e^{i \alpha} a_{j}: r \in[0, \infty), \mathbf{a}=\left(a_{j}\right)_{j=1}^{n} \in S^{n-1} \subset \mathbb{R}^{n} \subset \mathbb{C}^{n}\right\}
\end{aligned}
$$

where $\alpha$ is set to zero to give $L_{2}$.

So given a curve $\gamma$ in $\mathbb{C}$, we define a Lagrangian

$$
L_{\gamma}=\gamma \cdot S^{n-1}=\left\{z_{j}=\gamma a_{j}: \mathbf{a}=\left(a_{j}\right)_{j=1}^{n} \in S^{n-1} \subset \mathbb{R}^{n} \subset \mathbb{C}^{n}\right\} .
$$

Then $L_{2}$ is represented by $\gamma_{2}=[0, \infty) \subset \mathbb{C}, L_{1}$ by $\gamma_{1}=e^{i \alpha}[0, \infty) \subset \mathbb{C}$, and $L_{1} \cup L_{2}$ by the $\mathrm{V}$-shaped union of these curves.

In this notation the Lagrangian connect sum $L_{1} \# L_{2}$ is represented by any smoothing $\gamma=: \gamma_{1} \# \gamma_{2}$ of $\gamma_{1} \cup \gamma_{2}$ staying inside the cone $\left\{r e^{i \beta}: r>0, \beta \in[0, \alpha]\right\}$ which is $\gamma_{1} \cup \gamma_{2}$ outside a compact set, and a smooth curve cutting off the cone at the origin. (So here $\gamma$ is not a connect sum of the curves $\gamma_{i}$ in the topological sense; we only use the notation because the resulting Lagrangians are topological connect sums.)

We want to analyse the phase of such a connect sum; initially in the complex structure we picked on $T_{p} X \cong \mathbb{C}^{n}$ using the $x_{i}+i y_{i}$ coordinates and so, up to scale, $\left.\Omega\right|_{p}=d z_{1} \ldots d z_{n}$. Then the phase function of the Lagrangian $L_{\gamma}$ associated to a curve $\gamma$ is easily calculated to be $\theta\left(\gamma^{\prime}\right)+(n-1) \theta(\gamma)+N \pi$ for any $N \in \mathbb{Z}$ (where $\theta(z) \in(-\pi, \pi]$ is the phase of a complex number $\left.z=r e^{i \theta(z)}\right)$. Orienting $\gamma_{2}$ such that $\gamma_{2}^{\prime}$ is a positive real number, and choosing $L_{2}$ to have phase 0 , corresponds to choosing $N=0$ and so grading $L_{1}$ by

$$
(-\pi+\alpha)+(n-1) \alpha=n \alpha-\pi .
$$


In particular, choosing $\alpha=\pi / n$, and setting, for any $c>0$,

$$
\gamma_{c}=\left\{r e^{i \theta}: r^{n}=c \sin (n \theta), \theta \in(0, \pi / n)\right\}
$$

gives a SLag $L_{\gamma}$ which has a grading of phase identically zero, asymptotic to $L_{1}$ and $L_{2}$ at infinity, and this is precisely the local model of the example of Joyce, Harvey and Lawlor used so extensively in [Th].

While this is not strictly of the form $\gamma_{1} \# \gamma_{2}$ as defined above (it is only asymptotic to the $\gamma_{i}$, not equal outside a compact set), by taking $c$ small we can make it as close as we like to such a connect sum, all in the same hamiltonian deformation class, and the construction of Joyce is indeed a hamiltonian deformation of a connect sum as claimed in [Th].

We plot these SLag curves $\gamma_{c} \subset \mathbb{C}$ in Figure 1 as the light lines, converging as $c \rightarrow 0$ to the $\mathrm{V}$-shaped $\gamma_{1} \cup \gamma_{2}$ (with $\alpha=\pi / n$ ). Then the dark lines depict connect sums $L_{1} \# L_{2}$ for $\phi\left(L_{2}\right)=0$ and $\phi\left(L_{1}\right)= \pm \varepsilon$. If $\phi\left(L_{1}\right)<0$, the stable case as described in $[\mathrm{Th}]$, then we can choose the connect sum such that the phase of $L_{1} \# L_{2}$ varies monotonically between its values on $L_{1}$ and $L_{2}$, i.e. between $-\varepsilon$ and 0 .
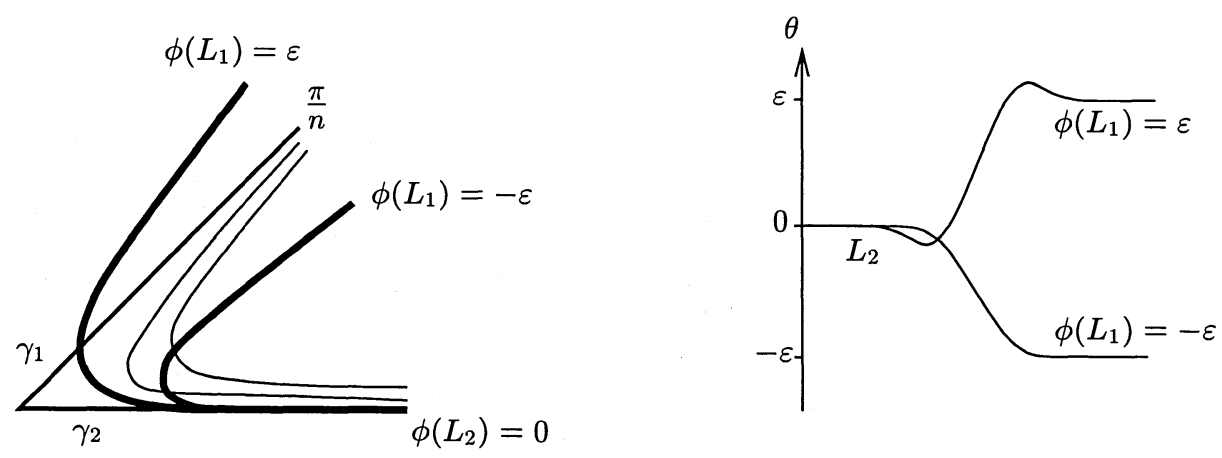

Figure 1: $\gamma_{1} \# \gamma_{2}$, and the resulting phase function $\theta_{L_{1} \# L_{2}}$, for $\phi\left(L_{1}\right)= \pm \varepsilon$

If, however, $\phi\left(L_{1}\right)>0$, the unstable case in [Th], then the phase of $L_{1} \# L_{2}$ must initially decrease to move away from $L_{2}$ before increasing to reach $L_{1}$ (i.e. $\gamma$ must cross the light lines one way then the other), giving a phase function which necessarily goes outside the range $(0, \varepsilon)$ (see Figure 1). This will be important to us later - under mean curvature flow we expect the phase function $\theta$ to evolve to a constant in the stable case (under the heat equation (2.5)) and to a Heaviside step function (with values 0 and $\varepsilon$ ) in the unstable case. This does not then contradict the maximum principle as the unstable case has the described non-monotonic phase. 
While this defines the symplectic connect sum in general by means of our Darboux chart, the analysis of phases depended on the choice of complex structure on $T_{p} X$, which may not have been the one restricted from the Calabi-Yau $X$. In the general case we can fix $\theta_{p}\left(L_{2}\right)=0$, without loss of generality, by rotating $\Omega$, and pick local complex coordinates $z_{i}=x_{i}+i y_{i}$ such that $\Omega_{p}=d z_{1} \ldots d z_{n}$ and, at the level of tangent spaces at $p$, (the tangent space to) $L_{2}$ is at $y_{i}=0 \forall i$. (The tangent space to) $L_{1}$ will be of the form

$$
L_{1}=\left\{z_{i}=r e^{i \alpha_{i}}\right\},
$$

for some $\alpha_{i}$ s that are no longer necessarily all the same in these coordinates. We are now connect summing Lagrangians of pointwise phase 0 and $\sum_{i=1}^{n}\left(\alpha_{i}\right)-\pi$ (compare (3.2)), but the resulting phase function will not be as simple as before - it is not pulled back from $\gamma$ but will vary over the $S^{n-1}$ fibres. Its average phase over the $S^{n-1} \mathrm{~s}$ will have a similar form to that in Figure 1, however, and in the case of all the $\alpha_{i}$ s being the same we get the earlier simpler picture.

The dependence of the hamiltonian deformation class of $L_{1} \# L_{2}$ on the choice of scale of the neck at each intersection point was described in ([Th] Section 4) (in particular if there is only one intersection point the class is uniquely defined). We should also point out that the graded connect sum (when it exists) is also independent of hamiltonian deformations of $L_{1}$ and $L_{2}$. While the $L_{i}$ s intersect transversely this is clear; we need only understand what happens in crossing the codimension one wall of Lagrangians intersecting in a double point (i.e. creating or cancelling two intersection points). But it will be clear from the definition of grading below that two such points must have grading differing by one, and so the connect sum along both of them cannot be graded (3.5).

\subsection{The grading on Floer cohomology.}

The Floer cohomology group $H F^{*}\left(L_{2}, L_{1} ; \mathbb{C}\right)$ [FO3] is the cohomology of a cochain complex made from a copy of $\mathbb{C}$ for each intersection point of two graded Lagrangians hamiltonian isotoped to intersect transversally. The differential is defined by counting holomorphic strips, with boundary in the Lagrangians, running from one intersection point to another. It is a symplectic refinement of the topological intersection theory of $L_{1}, L_{2}$, and as such is invariant only under hamiltonian deformations of the $L_{i}$. What is important to us is the grading of a particular transverse intersection point, as defined in [S2], [FO3]. 
While this can be defined completely topologically, it is most easily (and equivalently) defined via a complex structure. Again we work at the level of tangent spaces, pick local coordinates and, without loss of generality, take $L_{2}$ to have phase 0 and to be the $x$-axes: $L_{2}=\left\{y_{i}=0\right\}$. Write $L_{1}$ as

$$
L_{1}=\left\{z_{i}=r e^{i \alpha_{i}}\right\}
$$

where the $\alpha_{i} s$ are all in $(0, \pi)$. Then $\sum \alpha_{i}=\theta_{p}\left(L_{1}\right) \bmod \pi$ is the phase of $L_{1}$ up to multiples of $\pi$, and the following integer is the definition of the Floer index of the point $p$ :

$$
\operatorname{ind}_{p}\left(L_{2}, L_{1}\right):=\frac{1}{\pi}\left(\sum \alpha_{i}-\theta_{p}\left(L_{1}\right)\right) .
$$

Notice therefore that $\operatorname{ind}_{p}\left(L_{2}, L_{1}\right)+\operatorname{ind}_{p}\left(L_{1}, L_{2}\right)=n$. Applying the definition (3.4) to the connect sums defined in the last section (for which $\left.\theta_{p}\left(L_{1}\right)=\sum \alpha_{i}-\pi\right)$, we recover a result of Seidel [S2]:

$L_{1} \# L_{2}$ exists as a graded connect sum if and only if $\operatorname{ind}_{p}\left(L_{2}, L_{1}\right)=1$.

(The only if part follows from the independence of gradings and the Floer index from the complex structure; we may therefore pick the complex structure locally to have the form of the local model above.) Given $L_{1}$ there is at most one choice of the grading on $L_{2}$ such that $\operatorname{ind}_{p}\left(L_{2}, L_{1}\right)=1$ at all intersection points $p$, so that $L_{1} \# L_{2}$ can be graded.

In fact connect sums $L_{1} \# L_{2}$ whose own Floer cohomology is well defined [FO3] should correspond to Floer coclosed cochains, i.e. elements of $H F^{1}\left(L_{2}, L_{1}\right)$, mirror to the fact that extensions of sheaves $0 \rightarrow E_{1} \rightarrow E \rightarrow$ $E_{2} \rightarrow 0$ correspond to elements of $\operatorname{Ext}^{1}\left(E_{2}, E_{1}\right)$, as discussed in [Th].

We can also deal with the connect sums mentioned in [Th] which are relative versions of the above construction; $(n-r)$-dimensional connect sums carried out in a smooth family over an $r$-dimensional base. Then the same Floer index can be defined; there are now $r$ angles between the Lagrangians that are zero, and $(n-r)$ whose signs can be computed to get the Floer index. (The signs are constant over the family since the intersection of the Lagrangians $L_{1} \cap L_{2}$ fibres over the base of the family with fibres of constant dimension; an angle going to zero would cause a fibre dimension to increase.)

\section{Uniqueness.}

In finite dimensional symplectic quotient problems, convexity properties of the moment map prove uniqueness of its zeros (modulo the action of the real 
group) in a complexified group orbit. Translating this into our terms is not quite possible, because there are hamiltonian deformations of $L$ which are not given by the flow of a fixed hamiltonian on $L$. By this we mean $L_{0}, L_{1}$ are deformations given by a constant hamiltonian $h \in C^{\infty}\left(L_{0} ; \mathbb{R}\right)$ if the flow

$$
f_{t}: L \rightarrow W, \quad \frac{d f}{d t}=J \widetilde{d h}, \quad t \in[0,1],
$$

takes $L_{0}=f_{0}(L)$ to $L_{1}=f_{1}(L)$. All small deformations of a Lagrangian are of this form; for more general deformations we have to use a different proof of uniqueness of a SLag representative of a hamiltonian deformation class (Proposition 4.3 below), but for these constant hamiltonian deformations we describe the moment map proof to show how the formalism works.

Lemma 4.2. If two SLags $L_{0}, L_{1}$ are time-independent hamiltonian deformations of each other, in the sense above, then $L_{0}=L_{1}$.

Proof Without loss of generality we may take $\phi\left(L_{0}\right)=\phi\left(L_{1}\right)=0$. Then we compute, down the flow (4.1),

$$
\left.\frac{d}{d t} \int_{L} h \operatorname{Im} \Omega=\int h \mathcal{L}_{J \widetilde{d h}} \operatorname{Im} \Omega=\int h d(J \widetilde{d h}\lrcorner \operatorname{Im} \Omega\right)=\int \cos \theta d h \wedge * d h,
$$

where the last identity (equation (3.2) of [Th]) is an easy computation in local coordinates. (We have abused notation and written $\operatorname{Im} \Omega$ for $f_{t}^{*} \operatorname{Im} \Omega$.)

So for $\theta$ lying in $(-\pi / 2, \pi / 2)$ this is always strictly positive, but $\int_{L} h \operatorname{Im} \Omega$ is zero at $t=0,1$. Thus the two SLags must in fact coincide.

However, we must show that $\theta$ stays in this range if it starts in it, and deal with the case when it is not so bounded. The way to do this in fact proves the whole Lemma in one go anyway: pick a maximum $x \in L$ of $h$. Then by (2.4) $\dot{\theta}(x) \leq 0 \forall t$, but under the flow $\theta$ starts and ends at the same value (the cohomologically determined phase $\phi$ of the SLags). So $\Delta h=0$ and all the second derivatives $h_{x x} \leq 0$ in any direction $\partial_{x}$ vanish. Similarly then all third derivatives of $h$ must vanish (since we are at a maximum). Apply the same procedure to the second derivatives $h_{x x}$ of $h$ : $\dot{\theta}_{x x}=-\Delta_{L} h_{x x} \leq 0$ at $x$, since the other terms $\left[\partial_{x}^{2}, \Delta_{L}\right] h$ involve derivatives of the metric times third and lower order derivatives of $h$. Thus $h$ 's 4 th order derivatives vanish, and so on.

To get an integral form of this, to show that $h$ is in fact constant, it is enough to show that $h$ is constant in a small ball around any global maximum $x$ (with $h(x)=0$, without loss of generality). Consider geodesic balls $B_{r}$ of radius $r$ about $x$, and their boundary spheres $S_{r}$. Fix a standard unit-volume 
$(n-1)$-form $d \mu$ on the spheres $S_{r}$, so that the volume form induced by the metric is $\left.\partial_{r}\right\lrcorner$ vol $=A(r) d \mu$, where $r^{1-n} A(r)=c_{1}+O\left(r^{2}\right)$. For $r$ sufficiently small, $d / d r\left\{r^{1-n} A(r)\right\}$ is bounded by $c_{2} r$ (for some $c_{2}$ dependent only on the maximum of the curvatures of $L$ in a neighbourhood of $x$ over time $t \in[0, T]$ of the flow). Therefore

$$
\frac{d}{d r}\left\{\frac{\int_{S_{r}} h A(r) d \mu}{r^{n-1}}\right\}=r^{1-n} \int_{S_{r}} h_{r} A(r) d \mu+e,
$$

where $|e| \leq c_{3} r^{2-n}\left|\int_{S_{r}} h A(r) d \mu\right|$. Integrating over $r \in(0, R)$ for $R$ sufficiently small, and using the divergence theorem,

$$
f(R):=R^{1-n} \int_{S_{R}} h A(R) d \mu=\int_{0}^{R} r^{1-n}\left(\int_{B_{r}} \Delta h \mathrm{vol}\right) d r+E,
$$

where $|E| \leq c_{3} \int_{0}^{R}|r f(r)| d r$. Since $\Delta h=\dot{\theta}$, and $\int_{0}^{T} \dot{\theta} d t=0$ (where the hamiltonian deformation is over time $t \in[0, T]$, and $\theta$ starts and ends at the same value), we see that

$$
\int_{0}^{T}|f(R)| d t \leq c_{3} \int_{0}^{T} \int_{0}^{R}|r f(r)| d t d r
$$

for all small $R$. From this it follows that $f(r) \equiv 0$. That is, the average value of $h \leq 0$ over all small spheres surrounding $x$ (averaged over time as the metric on $L$ varies) is zero, and $h$ must be identically zero in a neighbourhhod of $x$.

However, we can do better by mirroring the algebro-geometric argument that a non-zero map between stable bundles of the same slope is an isomorphism, using the grading on Floer cohomology (3.4). This will appear to be slightly magical; the crux of the argument is the hamiltonian isotopy invariance of Floer cohomology, provided by precisely the holomorphic discs in the theory about which we have had so little to say. Again we work in $n$ dimensions.

Theorem 4.3. Pick a connected graded Lagrangian $L$ whose obstructions [FO3] to the existence of its Floer cohomology vanish, and whose second Stieffel-Whitney class $w_{2}$ is the restriction of a class $\in H^{2}(X ; \mathbb{Z} / 2)$ on the whole manifold (for instance if $L$ is spin).

Then there can be at most one smooth special Lagrangian in the hamiltonian deformation class of $L$. 
In particular, SLag homology spheres are unique in their hamiltonian deformation class in dimension 3 and above.

Proof Since Floer cohomology is independent of hamiltonian deformations [FO3], any two SLags $L_{1}, L_{2}$ in this same hamiltonian deformation class satisfy

$$
H F^{0}\left(L_{1}, L_{2}\right)=H^{0}\left(L_{1} ; \mathbb{C}\right)=\mathbb{C},
$$

given that the zeroth order piece of $H^{*}(L)$ survives in $H F^{*}(L, L)$ for $L$ with Maslov class zero ([FO3] Theorem E 1.7.4). Thus there must be at least one intersection point $p$ of $L_{1}$ and $L_{2}$.

We first want to show that the (constant) phases of the $L_{i}$ are the same; all we know a priori is that they differ by $r \pi$ for some $r \in \mathbb{Z}$. Using a hamiltonian perturbation we may assume then that there is at least one transverse intersection between the $L_{i}$ s of Floer index 0 , with the phases of the $L_{i}$ at this point differing by $r \pi+\varepsilon$. Thus writing, locally, $L_{2}$ as the graph in $T^{*} L_{1}$ of $d f, f$ has Morse index $r$ at the intersection point, so $r \geq 0$. Similarly there is a point of Floer index $n$ (i.e. a point of Floer index 0 when the roles of $L_{1}$ and $L_{2}$ are reversed) which must correspond locally to $d$ of a function of Morse index $n+r$; so $r \leq 0$ also. Therefore $r=0$ as required.

So we have SLags $L_{1}, L_{2}$ of the same pointwise phase with at least one intersection point which, if isolated, must have Floer index (3.4) zero. Thus $\theta_{p}=0$ in (3.4), and the definition (3.4) of the index is therefore always positive (in fact between 0 and $n$ ), and zero only if the relative angles $\alpha_{i}=$ $0 \forall i$. So the $L_{i}$ are tangent at $p$.

So there is no isolated transverse intersection point of Maslov class zero. In fact, working in a small neighbourhood of the intersection, we may choose coordinates such that $L_{1}$ is the graph in $T^{*} L_{2}$ of a closed one-form $\sigma$ on $L_{2}$ which is also coclosed in a certain metric on $T^{*} L_{2}$ in a first order infinitesimal neighbourhood of $L_{2}$ (coclosedness is the special Lagrangian condition). In this small open set, write $\sigma=d f$, so that $d^{*} d f=0$ and $f$ is harmonic; thus by the maximum principle, it has no local maxima or minima. We want to show that $f$ is in fact constant. The Floer index (3.4) of intersection points $d f=0$ now reduces to the Morse index of $f$ at isolated critical points, but we also have to deal with degenerate critical points of $f$. Assuming for a contradiction that the critical set of $f$ is not all of $L_{2}$, we may perturb $f$ inside any connected component of a small neighbourhood of its critical set such that its value is unchanged on the boundary, where it attains its global maximum and minimum, and is Morse in the interior. (That we may take the extrema to be on the boundary is the key point and a consequence of the maximum principle.) We can then perturb $f$ further to arrange its index 1 
critical points to be lower (with respect to $f$ ) than all higher index points (by general position arguments [Mi] Theorem 4.8) and then cancel any local minima with them ([Mi] Theorem 8.1). (There must be index 1 critical points if there are any interior minima, by connectivity of our neighbourhood.)

The upshot is a hamiltonian perturbation of $L_{1}$, using this new function, with no Floer index zero intersection points with $L_{2}$. Thus $H F^{0}\left(L_{1}, L_{2}\right)=0$, a contradiction, so in fact $f$ was locally constant and $L_{1}=L_{2}$.

The final statement follows from the fact that the obstructions of [FO3] live in $H^{2}(L)$, and homology spheres are spin.

As Donaldson pointed out, this proof is similar in flavour to proofs of the Arnold conjecture. If the local situation (of all hamiltonian deformations coming from a fixed function) held globally, the proof would be 'trivial', i.e. that of Lemma 4.2 above. Even more simply, if one SLag is a graph in the cotangent bundle of another, we reduce the problem to the uniqueness of harmonic functions of integral zero on $L$, i.e. to $H^{0}(L ; \mathbb{C})=\mathbb{C}$. To extend this argument globally we need to replace de Rham cohomology $H^{0}(L ; \mathbb{C})$ by Floer cohomology $H F^{0}(L, L ; \mathbb{C})$.

\section{Analogues of some properties of sheaves.}

In this section we discuss more properties of (S)Lags that mirror those of holomorphic vector bundles on Calabi-Yau manifolds. As they rely heavily on Floer cohomology arguments, many of the topics are necessarily treated informally and unrigorously for now.

\subsection{Twisting by line bundles.}

Any coherent sheaf can be twisted by a sufficiently positive line bundle $\mathscr{O}(N)$ so that it has sections; equivalently there are homomorphisms to the bundle from any sufficiently negative line bundle. If the sheaf has global support, this homomorphism is injective, exhibiting $E$ as an extension

$$
0 \rightarrow \mathscr{O}(-N) \rightarrow E \rightarrow Q \rightarrow 0
$$

One test of our notion of subobject of Lagrangians (in terms of connect sums), then, is that there should be appropriate connect sums mirroring this extension.

A line bundle $\mathcal{L}$ defines a spherical object [ST] of the derived category of sheaves on a Calabi-Yau manifold $X$; that is $\operatorname{Ext}^{i}(\mathcal{L}, \mathcal{L})=H^{0, i}(X) \cong$ 
$H^{*}\left(S^{n} ; \mathbb{C}\right)$ is $\mathbb{C}$ in dimensions 0 and $n$, and zero otherwise. These should be mirror to Lagrangian homology spheres; we will consider only spheres here so that we can use the graded Dehn twists [S2] around them. Negativity compared to some other Lagrangian may not make sense in general (intuitively, the Lagrangian might be mirror not to a sheaf but to an object of the derived category with Homs in negative degrees, etc.) but instead we can consider only those spheres $L$ with only degree zero intersection points (3.4) with a fixed Lagrangian $L^{\prime}$.

Then it is indeed true that we can exhibit $L$ as a subobject of $L^{\prime}$ : denoting by $T_{L}$ the (graded) symplectic Dehn twist about $L$, simply note that

$$
L^{\prime} \approx T_{L}^{-1} T_{L} L^{\prime} \approx L \#\left[L^{\prime} \#(L[1])\right]
$$

expresses $L^{\prime}$ as a connect sum of $L$ and something else. These relations can be shown by grading similar results in [S1]. In general this will not destabilise $L^{\prime}$ due to the phase of $L$ being so negative.

\subsection{Stability of (S)Lags.}

It is usual in correspondences between stable objects in algebraic geometry and solutions of the corresponding moment map PDE for one direction of the correspondence to be reasonably straightforward to prove, namely that objects which satisfy the PDE are stable.

While we cannot prove this for SLags, we can show, for SLags satisfying Floer cohomology restrictions as in Theorem 4.3 (in particular for spheres), that they cannot be destabilised by other SLags. (To test for stability of sheaves it is sufficient to test only with stable subsheaves; if the conjecture of [Th] is true then similarly we could test for stability of Lagrangians by connect summing only SLags; this would then be enough to prove the general stability of SLags.)

The idea is that if $\phi\left(L_{1}\right)>\phi(L)$, with both $L_{1}$ and $L$ SLags, then the Floer index of any intersection point of $L_{1}$ and $L$ is strictly positive (3.4), almost by definition. But if $L_{1}$ were to destabilise $L$, i.e. $L=L_{1} \# L_{2}$ for some $L_{2}$, then there should be canonical morphisms $H F^{0}\left(L_{1}, L\right) \neq 0$ and $H F^{0}\left(L, L_{2}\right) \neq 0$, a contradiction.

The morphism from $L_{1} \# L_{2}$ to $L_{2}$, by which we mean an element of

$$
H F^{0}\left(L_{1} \# L_{2}, L_{2}\right),
$$

can be described as follows (the element of $H F^{0}\left(L_{1}, L_{1} \# L_{2}\right)$ is similar). We use the description of the connect sum in Section 3. Choose a Morse function 
$f$ on $L_{2}$ which has local maxima at intersection points $p$ with $L_{1}$, and in local Darboux charts as in Section 3, is pulled up from a linear function on $\gamma_{2}$. Let the function have a unique local minimum elsewhere on $L_{2}$, and now use this to hamiltonian deform $L_{2}$ off $L_{1} \# L_{2}$. By construction $L_{1}$ and $L_{1} \# L_{2}$ now intersect at the critical points of $f$ only, with Floer index the Morse index of $f$. In terms of Figure 1, as $f$ has a maximum on $\gamma_{2}$ at the vertex of $\gamma_{2}$, it defines a hamiltonian deformation of $\gamma_{2}$ downwards, away from the connect-sum neck. As $L_{2}$ only intersects $L_{1}$ near these connect-sum necks, we can make our charts small enough that $L_{2}$ now only intersects $L_{1} \# L_{2}$ where its hamiltonian deformation intersects the old $L_{2}$, i.e. at the critical points of $f$.

We now have a unique index zero point of $\left(L_{1} \# L_{2}\right) \cap L_{2}$ at the unique local minimum of $f$. What we require is that this survives in the passage to cohomology of the cochain complex to give $H F^{0}\left(L_{2}, L_{1} \# L_{2}\right) \cong \mathbb{C}$. For instance, if there are no index one points (i.e. $f$ is a Morse function with only minima and index $\geq 2$ critical points) then this will clearly be the case. More generally there is a spectral sequence analogous to Poźniak's [P] with

$$
\operatorname{coker}\left\{\bigoplus_{i} \mathbb{C}_{p_{i}}[-n] \rightarrow H^{*}\left(L_{2}\right)\right\} \Longrightarrow H F^{*}\left(L_{1} \# L_{2}, L_{2}\right)
$$

(with a certain bigrading) converging to $H F^{*}\left(L_{1} \# L_{2}, L_{2}\right)$. Here the notation means that a copy of $\mathbb{C}$ is mapped to $H^{n}\left(L_{2}\right)$ (i.e. it is in degree $n$ ) for every intersection point $p_{i}$ via the Morse theory for $f$ (whose maxima are at the $\left.p_{i}\right)$. Therefore the degree zero part also survives if, for instance, $H^{1}\left(L_{2}\right)=0$. If $L_{2}$ is a sphere we can proceed more directly by applying Seidel's exact sequence [S3].

Using similar methods on Lagrangians rather than SLags, we can cut down on the number of possible destabilising Lagrangians $L_{1}$ we must check to conclude that a given $L$ is stable, rather analogously to only checking for subsheaves of vector bundles amongst those of lower rank. There are no morphisms (non-zero elements of $H F^{0}\left(L_{1}, L\right)$ ) if the phase of $L_{1}$, at an intersection point $p$, is greater than that of $L$; the Floer index at $p$ is strictly positive. So for $L_{1}$ to destabilise $L$ (and so $H F^{0}\left(L_{1}, L\right) \neq 0$ for Lagrangians satisfying the same conditions as above and in (4.3), e.g. homology spheres) we must have

$$
\inf _{x \in L_{1}} \theta_{L_{1}}(x)<\sup _{x \in L} \theta_{L}(x),
$$

and in fact the corresponding phase inequality at each point of intersection. 
Thus we do not have to check all Lagrangians $L_{1}, L_{2}$ for the stability of $L^{\prime}$ in [Th], just those whose phase function satisfies

$$
\inf _{x \in L_{1}} \theta_{L_{1}}(x) \leq \sup _{x \in L} \theta_{L}(x) \quad \text { and } \quad \sup _{x \in L_{2}} \theta_{L_{2}}(x) \geq \inf _{x \in L} \theta_{L}(x),
$$

where we can in fact replace the left hand sides of these inequalities by the inf (respectively sup) over all Lagrangians in the same hamiltonian deformation class.

Assuming the conjecture in [Th], so that we need only check SLag destabilisers to show that stability of $L_{0}$, we are reduced to checking for destabilising subobjects amongst those $L_{1}, L_{2}$ whose homology classes sum to $\left[L_{0}\right]$ and satisfy

$$
\sup _{L \approx L_{0}}\left(\inf _{x \in L} \theta_{L}(x)\right) \leq \phi\left(L_{1}\right) \leq \phi\left(L_{2}\right) \leq \inf _{L \approx L_{0}}\left(\sup _{x \in L} \theta_{L}(x)\right) .
$$

\subsection{A Jordan-Hölder decomposition for Lagrangians.}

In order to understand limits of mean curvature flow it will be useful to have the following concept; an analogue for Lagrangians of the Jordan-Hölder filtration of sheaves (see [HuL] 1.5, for instance).

Definition 5.3. Given two graded Lagrangians $L_{1}, L$, write $L_{1} \leq L$ if there exists a graded Lagrangian $L_{1}^{\prime}$ such that $L \approx L_{1} \# L_{1}^{\prime}$. We then also write $L / L_{1}$ for $L_{1}^{\prime}$, and say that $L_{1}$ is a subobject of $L$.

A Jordan-Hölder filtration of $L$ is a sequence of graded Lagrangians $L_{i}$ such that

$$
L_{1} \leq L_{2} \leq \ldots \leq L_{k}=L,
$$

and $L_{i}^{\prime}:=L_{i+1} / L_{i}$ is stable. The Jordan-Hölder decomposition of $L$ is the the singular union

$$
L_{1} \cup\left(L_{2} / L_{1}\right) \cup \ldots \cup\left(L / L_{k-1}\right) .
$$

In sheaf theory the Jordan-Hölder filtration need not be unique, but the decomposition is. For smooth connected Lagrangians, with connected $L_{i}$ for all $i$, however, we expect the filtration to be unique too; the difference is essentially that while direct sum is an operation on bundles, we are proposing that its mirror is the (singular) union of Lagrangians, and this cannot give a smooth Lagrangian if there is non-zero Floer cohomology between the two Lagrangians. 
If we assume the conjectures of [Th] and Section 7 below, and the properties of Floer cohomology [FO3] for all of the above Lagrangians (e.g. if they are homology spheres), we can demonstrate the existence and uniqueness of the Jordan-Hölder filtration for a Lagrangian $L$ whose phase function of $L$ satisfies $\sup \theta_{L}-\inf \theta_{L}<\pi$.

Without loss of generality we may assume (by rotating $\Omega$ ) that $\theta$ lies between $\pi / 2-\varepsilon$ and $-\pi / 2+\varepsilon$, for some $\varepsilon>0$. By the inequality (5.2) above, then, any $L_{1} \# L^{\prime}$ destabilising it will satisfy $\phi\left(L_{1}\right), \phi\left(L^{\prime}\right) \in(-\pi / 2+$ $\varepsilon, \pi / 2-\varepsilon)$.

We choose such an $L_{1}$ of maximal phase, and, amongst other such $L_{1} \mathrm{~s}$ of the same phase, minimal $\int_{L_{1}} \operatorname{Re} \Omega$ (for the purposes of this proof we will call this quantity cohomological volume). This still need not specify $L_{1}$ uniquely though.

We claim that such an $L_{1}$ must be stable by construction. Any subobject of $l \leq L_{1}$ would also be a subobject of $L$ and so by the construction of $L_{1}$ must either have smaller phase, which is not possible since it destabilises $L_{1}$, or equal phase and greater or equal cohomological volume. But $L_{1}=l \# l^{\prime}$, where $l^{\prime}=L_{1} / l$ has phase $\phi\left(l^{\prime}\right)=\phi\left(L_{1}\right) \in(-\pi / 2, \pi / 2)$ and so positive cohomological volume $\int_{l^{\prime}} \operatorname{Re} \Omega$. So the complex numbers

$$
\int_{L_{1}} \Omega=\int_{l} \Omega+\int_{l^{\prime}} \Omega
$$

all have positive real part, implying that the cohomological volume of $l$ is strictly less that that of $L_{1}$, a contradiction.

We then apply the same procedure to $L^{\prime}$, producing an $L_{2} \hookrightarrow L^{\prime}$, and so on. By construction $\phi\left(L^{\prime} / L_{2}\right) \leq \phi\left(L^{\prime}\right) \leq \phi(L)<\pi / 2-\varepsilon$, and there is a canonical morphism (5.1) in $H F^{0}\left(L, L^{\prime} / L_{2}\right) \neq 0$, making $\phi\left(L^{\prime} / L_{2}\right) \geq$ $\inf _{L} \theta_{L}>-\pi / 2+\varepsilon$ by (5.2).

Thus, inductively, we get the same inequalities at each stage, and the cohomological volume of $L^{\prime}$ decreases strictly with each decomposition $L \approx$ $L_{1} \# \ldots \# L_{n} \# L^{\prime}$. The cohomological volume of any $l$ with phase $\phi(l) \in$ $(-\pi / 2+\varepsilon, \pi / 2-\varepsilon)$ is greater than (or equal to in the SLag case) $\cos (\pi / 2-$ $\varepsilon) \int_{l}$ vol, by (1.1), where vol is its Riemannian volume form. This is bounded below above zero, so the process can have at most a finite number of steps.

This gives us the Jordan-Hölder filtration; next we consider uniqueness when the $L_{i}$ s are connected (assuming the conjectures of [Th] and Section 7 and some Floer cohomology). Suppose that $L_{1}^{\prime} \leq L_{2}^{\prime} \leq \ldots \leq L$ is another such connected decomposition where we take the $L_{i}^{\prime}$ s to be SLag assuming the conjecture of [Th]. If $H F^{0}\left(L_{1}^{\prime}, L_{1}\right) \neq 0$ then by the proof of Theorem 
4.3 (which applies as $L_{1}^{\prime}$ and $L_{1}$ have the same phase), $L_{1}$ and $L_{1}^{\prime}$ are equal, and we pass to $L_{2}$.

If, however, $H F^{0}\left(L_{1}^{\prime}, L_{1}\right)=0$, then we claim that $H F^{0}\left(L_{1}^{\prime}, L / L_{1}\right) \neq$ 0 . Again this should follow from standard facts about Floer cohomology, in particular a long exact sequence $H F^{*}\left(L_{1}^{\prime}, L_{1}\right) \rightarrow H F^{*}\left(L_{1}^{\prime}, L\right) \rightarrow$ $H F^{*}\left(L_{1}^{\prime}, L / L_{1}\right) \rightarrow H F^{*+1}\left(L_{1}^{\prime}, L_{1}\right)$. For $L / L_{1}$ a sphere this is Seidel's exact sequence ([S3] Theorem 3.3), and in general one can establish it at the level of chains by good choices of hamiltonian perturbations as in Section 5.2; as usual the problem is in controlling the differential, i.e. holomorphic discs.

Assuming this we may pass to $L_{2}$; inductively we eventually obtain that $L_{1}^{\prime}$ is isomorphic to one of the graded pieces $L_{i+1} / L_{i}$ of the original filtration, and is a subobject of $L_{i+1}$ but not of $L_{i}$. But this gives us a contradiction (in contrast to the sheaf analogue), since we have that both $L_{i+1} \approx L_{i} \# L_{1}^{\prime}$ and $L_{1}^{\prime}$ is a subobject of $L_{i+1}$. The first condition ensures that there are representatives of the hamiltonian deformation classes such that $L_{i+1}$ and $L_{1}^{\prime}$ have no index $n$ intersection points by the construction of (5.1), so that $H F^{n}\left(L_{i+1}, L_{1}^{\prime}\right)=0$. But this is $H F^{0}\left(L_{1}^{\prime}, L_{i+1}\right)^{*}$, which cannot vanish by the second condition. (It is here we use the connectivity condition, i.e. that the connect sum $L_{i+1}=L_{1}^{\prime} \#\left(L_{i+1} / L_{1}^{\prime}\right)$ is not a trivial disjoint union. Without the connectivity condition the usual proof (e.g. [HuL] 1.5) that the JordanHölder decomposition (rather than filtration) of sheaves is unique applies to Lagrangians, now that we have proved or assumed all (the mirror analogues of) the algebraic facts used for sheaves in terms of Floer cohomology instead of Exts.

So in the simplest case of instability, such as the example of Joyce considered in [Th], where $L=L_{1} \# L_{2}$ is the only relevant decomposition of $L$ with $\phi\left(L_{1}\right) \geq \phi\left(L_{2}\right)$, the Jordan-Hölder decomposition (5.4) would be simply $L_{1} \cup L_{2}$ (where the $L_{i}$ are SLag representatives of their classes). This, like all such decompositions, is in the closure of the hamiltonian deformation orbit of $L$ while not being in the orbit itself.

This should have relevance to the Schoen-Wolfson programme [SW] to find canonical representatives (in a fixed hamiltonian deformation class) of Lagrangian homology classes using volume minimisers and so SLags (they do not use a flow, but regularity results to study minimising currents). Our conjecture (as in [Th] and later in Section 7) should either provide a unique SLag in a hamiltonian deformation class, or a number of SLags in a JordanHölder decomposition.

For instance in the example above of $L_{1} \# L_{2}$ in 2 dimensions we would produce SLags in the classes of $L_{1}$ and $L_{2}$, but we could also form $L_{2} \# L_{1}$; 
this could then be stable (it is no longer destabilised by either of the $L_{i}$; if the phases of the $L_{i}$ are sufficiently close one can show that in fact nothing else destabilises it either) and we should recover a SLag in this class (and so in the same homology class in two dimensions).

Since in two dimensions SLags are just holomorphic curves with respect to a different complex structure, this places heavy restrictions on stability. Take the $L_{i}$ above to be spheres in $K 3$ surfaces. Then any holomorphic sphere is unique in its homology class (it has negative self intersection -2 , so does not lie in a pencil). Any other homologous hamiltonian deformation class must therefore be unstable. Good examples are provided by taking a stable (SLag/holomorphic) sphere, and applying the square of a Dehn twist $T_{L_{1}}^{2}$ to it; this preserves homology classes but can change hamiltonian deformation classes. If it does it should produce an unstable Lagrangian with copies of $L_{1}$ in its Jordan-Hölder decomposition; this happens in all simple cases. $L_{1} \# L_{2}$ is taken to $L_{2} \# L_{1}$, for instance; only one of these can be stable, the other having a Jordan-Hölder decomposition $L_{1} \cup L_{2}$ in the simplest case.

More generally, instead of studying the action on individual (S)Lags of symplectomorphisms like $T_{L_{1}}^{2}$ above, we could try to study them all at once by studying the Lagrangian graph of the symplectomorphism in $X \times X$, and its mean curvature flow. This looks for minimal energy representatives of the hamiltonian isotopy class of a symplectomorphism, and breaks graphs up into correspondences representing singular maps (birational maps in the hyperkähler case) with singularities concentrated in loci whose stability is affected by the symplectomorphism. For a Dehn twist $T_{L}$, for instance, we would expect to get the graph $\Delta$ of the identity, union $L \times L$. This also shows what the analogue of a Dehn twist $T_{L}$ should be when $L$ is not a sphere but a rational homology sphere (so that it is still spherical to complex coefficients, and so mirror to a twist on the derived category of sheaves on the mirror Calabi-Yau [ST]). Namely $\Delta \cup L \times L$ is a Lagrangian correspondence in $X \times X$ which should give an automorphism of the derived Fukaya category of $X$ (by the usual Fourier-Mukai-type construction) not induced by a symplectomorphism of $X$.

\section{An example: families of affine quadrics.}

Here we consider an example suggested to us by both Paul Seidel and Cumrun Vafa, used in [SV] and [KS]. Consider the affine algebraic variety $X^{n}$ 
given by

$$
\sum_{i=1}^{n} x_{i}^{2}=p(t)
$$

in $\mathbb{C}^{n} \times \mathbb{C}$, where $p$ is some polynomial in $t \in \mathbb{C}$ with only simple zeros. Denote by $\pi: X^{n} \rightarrow \mathbb{C}$ the projection to the $t$ coordinate. Here we use the Kähler structure restricted from $\mathbb{C}^{n+1}$, and the nowhere-zero holomorphic volume form given by taking the Poincaré residue ([GH] p 147) of the standard form $d x_{1 \ldots n} d t:=d x_{1} \ldots d x_{n} d t$ on $\mathbb{C}^{n+1} ;$ this can be written as

$$
(-1)^{n+i+1} \frac{\left.d x_{1 \ldots \hat{i} \ldots n} d t\right|_{X^{n}}}{2 x_{i}}=\frac{\left.d x_{1} \ldots d x_{n}\right|_{X^{n}}}{\dot{p}(t)}
$$

for any $i$ (so where $x_{i}=0 \forall i$ we can use the second expression). Here $\hat{\imath}$ means that we omit the $d x_{i}$ term from the wedge product. This is then not parallel, and the metric we have chosen is not the Ricci-flat one. Nonetheless it is a good explicit testing ground for the conjecture; we can still define $\theta$ as the phase of $\left.\Omega\right|_{L}$ and SLags as having constant phase, of course we then use flow by the $J \widetilde{d \theta}$ vector, rather than mean curvature flow in this metric. While the two flows are similar and would be the same in the Ricci-flat metric, only the former has SLags as its stationary points (for the latter we get minimal submanifolds, which in this metric are not quite SLag). As Edward Goldstein pointed out to us, the $J \widetilde{d \theta}$ flow is the gradient flow of the weighted volume functional $\int_{L}|\Omega|$ vol instead of $\int_{L}$ vol; everything proceeds analogously to before on weighting all vols by $|\Omega|$, as we shall see.

Each smooth fibre over $t \in \mathbb{C}$ is an affine quadric with a natural Lagrangian $S^{n-1}$ 'real' slice, namely the intersection of the fibre with the slice

$$
x_{i} \in \sqrt{p(t)} \mathbb{R} \quad \forall i .
$$

It is invariant under the obvious $O(n)$ action on $X^{n}$, and is the vanishing cycle of every singular fibre (i.e. the fibres over the roots of $p$ ). Therefore any path $\gamma: I \rightarrow \mathbb{C}(I \ni u$ being some interval in $\mathbb{R})$ from one zero of $p$ to another lifts to give a canonical $O(n)$-invariant Lagrangian $n$-sphere $\gamma^{n}$, $S^{n-1}$-fibred over $\gamma$ except at the endpoints where it closes up. Also, any vector $\gamma^{\prime} \partial_{t}$ in the base $\mathbb{C} \ni t$ lifts canonically to a vector

$$
\gamma^{\prime}\left(\partial_{t}+\frac{\dot{p}}{2 p} \sum x_{i} \partial_{x_{i}}\right)
$$

tangent to the infinitesimal Lagrangian $\gamma^{n}$ lying above $\gamma^{\prime}$. Here ' denotes $d / d u$. Note that $\gamma^{1}$ is a closed curve double covering $\gamma$, branched over $\gamma^{\prime}$ s endpoints. We will use this curve $\gamma^{1}$ later to study $\gamma^{n}$. 
The phase function $\theta$ on $\gamma^{n}$ is also $O(n)$-invariant and so a function of $t \in \mathbb{C}$ which we may calculate at $x_{1}=\sqrt{p(t)}, x_{i}=0 \forall i \geq 2$. Choosing a basis of tangent vectors to $\gamma^{n}$ at this point,

$$
\gamma^{\prime}\left(\partial_{t}+\frac{\dot{p}}{2 p^{1 / 2}} \partial_{x_{1}}\right), \sqrt{p} \partial_{x_{2}}, \ldots, \sqrt{p} \partial_{x_{n}},
$$

wedging them together and evaluating against the $(n, 0)$-form $(6.1)$ gives

$$
\gamma^{\prime} \frac{\dot{p}}{2 p^{1 / 2}} \frac{(\sqrt{p})^{n-1}}{\dot{p}}=\frac{1}{2} \gamma^{\prime} p^{n / 2-1} .
$$

Therefore the phase function on $\gamma^{n}$ is given by

$$
\theta:=\theta\left(\gamma^{n}\right)=\theta\left(\gamma^{\prime}\right)+\left(\frac{n}{2}-1\right) \theta(p(\gamma)),
$$

where $\theta\left(\gamma^{\prime}\right)$ is the usual angle of the path $\gamma$, and $\theta(p)$ is the phase of the complex number $p$ evaluated at $t=\gamma$. So

$$
d \theta=\left(\frac{d \theta\left(\gamma^{\prime}\right)}{d u}+\left(\frac{n}{2}-1\right) \frac{d \theta(p)}{d u}\right) d u
$$

where $d u$ is the pullback to $\gamma^{n}$ under the projection $\pi$ of the corresponding 1-form on $\gamma(I) \subset \mathbb{C}$.

Using the metric and the orthogonal basis (6.3) we see that

$$
\widetilde{d u}=\frac{\gamma^{\prime}\left(\partial_{t}+\frac{\dot{p}}{2 p^{1 / 2}} \partial_{x_{1}}\right)}{\left|\gamma^{\prime}\left(\partial_{t}+\frac{\dot{p}}{2 p^{1 / 2}} \partial_{x_{1}}\right)\right|^{2}}
$$

at the point $x_{1}=\sqrt{p(t)}, x_{i}=0 \forall i \geq 2$.

Therefore, by $O(n)$-invariance and the holomorphicity of the projection $\pi, J \widetilde{d \theta}$ is the canonical lift (6.2) of

$$
\begin{aligned}
& \frac{d\left(\theta\left(\gamma^{\prime}\right)+\left(\frac{n}{2}-1\right) \theta(p)\right)}{d u} \frac{\pi_{*}\left[J \gamma^{\prime}\left(\partial_{t}+\frac{\dot{p}}{2 p^{1 / 2}} \partial_{x_{1}}\right)\right]}{\left|\gamma^{\prime}\right|^{2}\left(1+|\dot{p}|^{2} / 4|p|\right)} \\
& =\frac{\frac{1}{\left|\gamma^{\prime}\right|} \frac{d}{d u}\left(\theta\left(\gamma^{\prime}\right)+\left(\frac{n}{2}-1\right) \theta(p)\right)}{1+|\dot{p}|^{2} / 4|p|} i \frac{\gamma^{\prime}}{\left|\gamma^{\prime}\right|} \partial_{t} .
\end{aligned}
$$

Denoting by $\mathbf{t}=\partial_{u} /\left|\gamma^{\prime}\right|=\gamma^{\prime} \partial_{t} /\left|\gamma^{\prime}\right|$ and $\mathbf{n}=i \mathbf{t}$ the unit tangent and normal vectors to $\gamma$ at a point $\gamma(u)$, the above is

$$
\frac{\mathbf{t}\left[\theta\left(\gamma^{\prime}\right)+\left(\frac{n}{2}-1\right) \theta(p)\right]}{1+|\dot{p}|^{2} / 4|p|} \mathbf{n} .
$$


By the Cauchy-Riemann equations for the holomorphic function $\log p=$ $\log |p|+i \theta(p), \mathbf{t} \theta(p)=-\mathbf{n} \log |p|$, so that our flow is the lift to $X^{n}$ of the flow of $\gamma$ with vector

$$
V^{n}=\frac{1}{1+|\dot{p}|^{2} / 4|p|}(\mathrm{MCV}+(1-n / 2) \mathbf{n}(\log |p|) \mathbf{n}),
$$

where MCV is the usual mean curvature vector of $\gamma$ in the flat metric on $\mathbb{C}$.

So we can reduce studying our flow to studying the flow of a curve $\gamma$ with fixed endpoints (at zeros of $p$ ), under the above vector field. We would like to relate this to mean curvature flow of $\gamma \subset \mathbb{C}$ in a different metric, and also to both our flow and the mean curvature flow for the double $\gamma^{1}$ of $\gamma$ in the double cover $X^{1}$ of $\mathbb{C}$ branched over the zeros of $p$. The advantage of this is that we now have a flow for a closed curve instead of a boundary value problem (but since the flow has $O(1)=\mathbb{Z} / 2$ symmetry it is equivalent to a flow of the original curve $\gamma$ with fixed endpoints). We need the following lemma.

Lemma 6.6. Let $\langle.,$.$\rangle be the standard metric on \mathbb{C}$, and $g$ a positive realvalued function on $\mathbb{C}$. Then with respect to the metric $g\langle.,$.$\rangle , the mean$ curvature vector of a curve $\gamma \subset \mathbb{C}$ is, in terms of the standard mean curvature vector MCV (and calculating the unit normal $\mathbf{n}$ in the standard metric),

$$
\frac{1}{g}\left(\mathrm{MCV}-\frac{1}{2} \mathbf{n}(\log g) \mathbf{n}\right) .
$$

Proof The endomorphism-valued 1-form $\Gamma$ defined by

$$
\Gamma_{X} Y=\frac{1}{2 g}((X g) Y+(Y g) X-\langle X, Y\rangle \widetilde{d g})
$$

is symmetric and so defines a torsion-free connection on $\mathbb{C}$. It is easily checked to be orthogonal with respect to the metric $g\langle.,$.$\rangle , and so gives its$ Levi-Civita connection $\nabla+\Gamma$ (where $\nabla$ is the usual connection on $\mathbb{C}$ ). Then $\left\langle\Gamma_{\gamma^{\prime}} \gamma^{\prime}, \mathbf{n}\right\rangle$ (where $\mathbf{n}$ is calculated in the original metric) is $-\frac{1}{2 g}\left|\gamma^{\prime}\right|^{2} \mathbf{n}(g)=$ $-\frac{1}{2}\left|\gamma^{\prime}\right|^{2} \mathbf{n}(\log g)$.

Since the unit normal to $\gamma$ in the new metric is $g^{-1 / 2} \mathbf{n}$, the new mean curvature vector is

$$
\frac{g\left\langle\gamma^{\prime \prime}+\Gamma_{\gamma^{\prime}} \gamma^{\prime}, g^{-1 / 2} \mathbf{n}\right\rangle}{g\left|\gamma^{\prime}\right|^{2}} g^{-1 / 2} \mathbf{n}=\frac{1}{g}\left(\mathrm{MCV}-\frac{1}{2} \mathbf{n}(\log g) \mathbf{n}\right),
$$

as claimed. 
Using this we can get a number of geometrically interesting flows which are equivalent to our original flow in $X^{n}$. Namely, using the result (6.5), the above Lemma, and the fact that locally (away from branch points) $X^{1}$ is conformally equivalent to $\mathbb{C}$ with its metric scaled by $g=1+|\dot{p}|^{2} / 4|p|$ (by holomorphicity and (6.2)), we can deduce the following.

Denote by $V^{n}$ the flow vector $\pi_{*} J \widetilde{d \theta}$ of the curve $\gamma \subset \mathbb{C}$ under our flow in $X^{n}$. Denote by $\mathrm{MCV}_{g}^{1}$ the flow vector of $\gamma \subset \mathbb{C}$ under mean curvature flow of $\gamma^{1}$ in $X^{1}$, with $X^{1}$ 's natural metric scaled by a $\mathbb{Z} / 2$-invariant function $g$ (and omit the $g$ in the notation if $g \equiv 1$ ). And denote by $\mathrm{MCV}_{g}$ the mean curvature vector of $\gamma$ in $\mathbb{C}$ with metric $g\langle.,$.$\rangle .$

Letting $\mathbf{n}$ be the unit normal to $\gamma$ calculated in the standard metric on $\mathbb{C}$, and letting $f=\frac{\mid p n^{n-1}}{|p|+|\dot{p}|^{2} / 4}$, we have the following relations between the various flows:

$$
V^{n}=f \cdot \mathrm{MCV}_{f}^{1}=f \cdot \mathrm{MCV}_{|p|^{n-2}},
$$

and

$$
V^{n}=\mathrm{MCV}^{1}-\left[\frac{1}{2}|p|^{2-n} \mathbf{n}(f)\right] \mathbf{n}=V^{1}-\frac{1}{2}(n-1) \frac{\mathbf{n}(|p|)}{|p|+|\dot{p}|^{2} / 4} \mathbf{n} .
$$

The problem with the first two is that on $\mathbb{C} \supset \gamma$ the flow is not parabolic, it has degeneracies at the end points. As $X^{1}$ is so closely modelled on $X^{n}$ (and is in fact canonically embedded in it), however, we might expect better on $X^{1}$. This is more or less true; the result is that writing (6.8) in terms of the unit normal $\mathbf{n}^{1}$ on $X^{1}$, we get

\section{Theorem 6.9.}

$$
V^{n}=\operatorname{MCV}^{1}-\frac{1}{2}(n-1) \mathbf{n}^{1}(\log |p|) \mathbf{n}^{1}+\frac{1}{2} \mathbf{n}^{1}\left(\log \left(|p|+|\dot{p}|^{2} / 4\right)\right) \mathbf{n}^{1} .
$$

The last term is bounded (as near a zero of $p, \dot{p} \neq 0$ by nondegeneracy of $p$ 's zeros) and so unimportant, we shall see, and the flow resulting from the first term is well understood. The second term is more curious; it is of the order of $\mathbf{t}^{1} \theta(p) \approx \mathbf{t}^{1} \theta\left(\left(\gamma^{1}\right)^{\prime}\right) / 2$ (where ${ }^{\prime}=\mathbf{t}^{1}$ denotes differentiation with respect to arclength on $\left.X^{1}\right)$ whenever we are close to a point where $\gamma$ emanates from a zero of $p$ (so that $p \approx C t$ and $\theta(p) \approx \theta(\gamma) \approx \theta\left(\gamma^{\prime}\right) \approx$ $\theta\left(\left(\gamma^{1}\right)^{\prime}\right) / 2$ ). (The last approximation is of course not valid if $\gamma$ simply passes close to a zero of $p$; then the equation blows up quickly as a glance at (6.5) shows, $\gamma$ flowing to this zero and breaking across it as discussed below; in the 
stable case we will be able to rule out this behaviour and need only consider $\gamma$ ending at the zero.)

But this is half the curvature of $\gamma^{1}$, so we get an approximation to the first term again, and something like mean curvature flow for $\gamma^{1} \subset X^{1}$. In fact in a small neighbourhood of (the double cover of) a zero of $p$, in coordinates $(x, y)$ in which $\gamma^{1}$ is a graph $y(x)$, the evolution PDE is of the general shape

$$
y_{t}=y_{x x}+\frac{y_{x}}{x}
$$

where by the $\mathbb{Z} / 2$-symmetry $\left.y_{x}\right|_{x=0}=0$, so the second term is approximately $y_{x x}$. So for some analysis we use this flow for $\gamma^{1}$, while for the rest we pass back to $n$-dimensions, and work with the phase function $\theta$ instead, giving a more standard (but $n$-dimensional) parabolic equation.

We first assert how the flow behaves, before proving it in the stable case in the next section (Theorem 7.6). Note that any deformation of $\gamma$ is a hamiltonian deformation of $\gamma^{n}$ (and SLag $\gamma^{n}$ s have no moduli) since the $\gamma^{n} \mathrm{~S}$ are spheres. We picture what happens in Figures 2 and 4 in the 2 and 3 dimensional cases respectively. The dots represents zeros of $p$ in both cases, and the epsilons and zeros are phases.
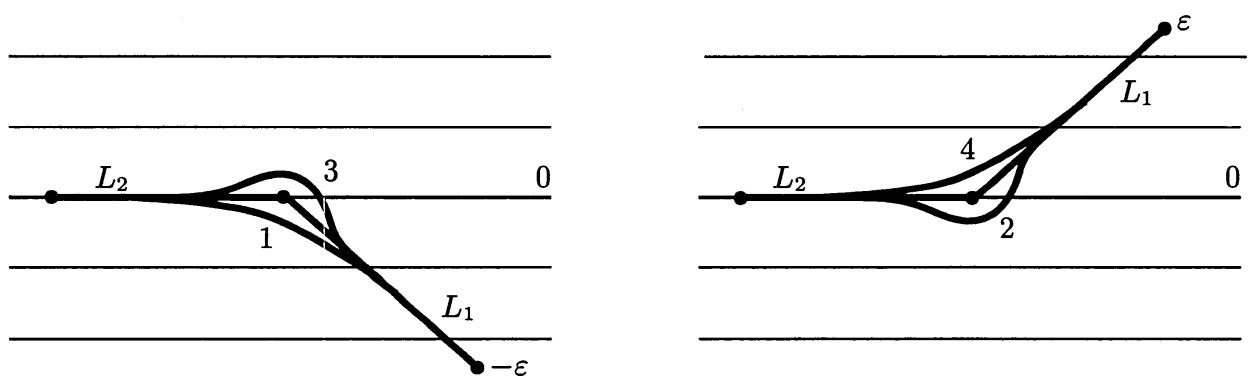

Figure 2: The two connect sums $L_{1} \# L_{2}(1,2)$ and $L_{2} \# L_{1}(3,4)$ in 2dimensions

In two dimensions the curves $\gamma$ whose Lagrangians $\gamma^{2}$ have constant phase are the straight lines, as can be seen from (6.4). Curves such as those marked 1 and 4 in Figure 2 flow towards a straight line (of some non-zero angle) corresponding to a SLag, whereas curve 2 flows up until it 'hangs' on a zero of $p$ (in finite time), where, on restarting the flow for 2 different curves, the separate flows form a kink and in the limit converge to destabilising SLags $L_{1}, L_{2}$ of different phases. These unions of SLags of different phases are still stationary for the volume functional (satisfying the second 
order variational equations, just not the first order SLag equations), and in fact are minimising in odd dimensions (the angle criterion $[\mathrm{N}],[\mathrm{L}]$ makes minimality of the singular union locally equivalent to the above destabilising phase condition; in even dimensions reversing the order of the Lagrangians reverses the inequality and the configuration is not minimal, just stationary).

Again we see how the phase or angle criterion comes to bear; curves 1 and 2 are in the same homology class, but the two different phase signs give very different results. As noted before in Figure 1, this is related to the necessity of the phase to vary non-monotonically to form the unstable connect sum; in Figure 3 we plot the phases of the two connect sums, and with dotted lines their limits under the heat flow (7.4) (this is the correct modification of (2.5) in the non Ricci-flat case).
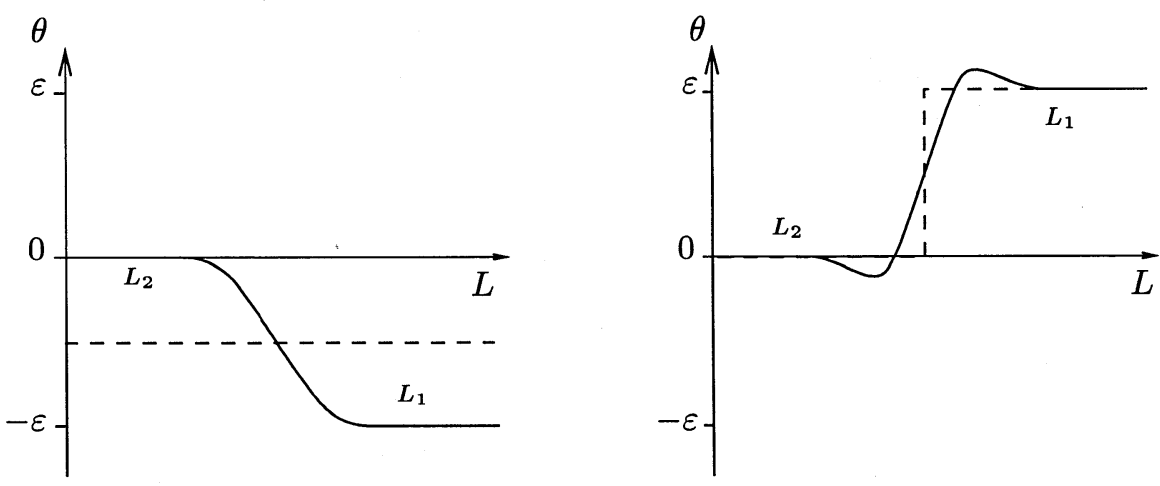

Figure 3: The phase $\theta_{L=L_{1} \# L_{2}}$ of (1) $\left(\theta_{L_{1}} \equiv-\varepsilon\right)$ and (2) $\left(\theta_{L_{1}} \equiv \varepsilon\right)$ respectively.

Drawing $\gamma$ the other way round the zero of $p$ gives something in the same homology class (the Dehn twist around the root of $p$ does not alter the homology class of the $S^{1}$ fibre over $\gamma$ ), which is the opposite connect sum discussed in $[\mathrm{Th}]$ - once the phase inequality becomes unstable for one connect sum it becomes stable for the other.

The two connect sums are related by monodromy, as in [Th]. Take a one parameter family of polynomials $p$ which rotates two zeros $z_{1}, z_{2}$ of $p$ around each other. Then under the resulting monodromy a curve joining $z_{1}$ to a third zero $z_{3}$ is taken from being 'above' $z_{2}$ to being below it, thus turning one connect sum into the other.

The three dimensional picture is similar. In Figure 4 we plot the lines corresponding to SLags of phase zero, and connect sums $L_{1} \# L_{2}$ for $\phi\left(L_{2}\right)=$ 
0 and $\phi\left(L_{1}\right)=\mp \varepsilon$ (curves 1 and 2). Again we see the same behaviour with the phases behaving as in the graphs in Figure 3 and the flow getting hung on a zero of $p$ in the unstable case, splitting the Lagrangian.

Reversing the order of the connect sum in this case involves taking the $S^{2}$ fibre once around the zero of $p$; this effects a Dehn twist, reversing its orientation. Thus although curve 3 appears to give a Lagrangian in the same homology class, it is not; the phase of $L_{1}$ once we have been round the root of $p$ has shifted by $\pi$ and we get the connect sum $L_{2} \#\left(L_{1}[-1]\right)$ discussed in [Th]. As is also discussed there, this can be seen to be unstable.

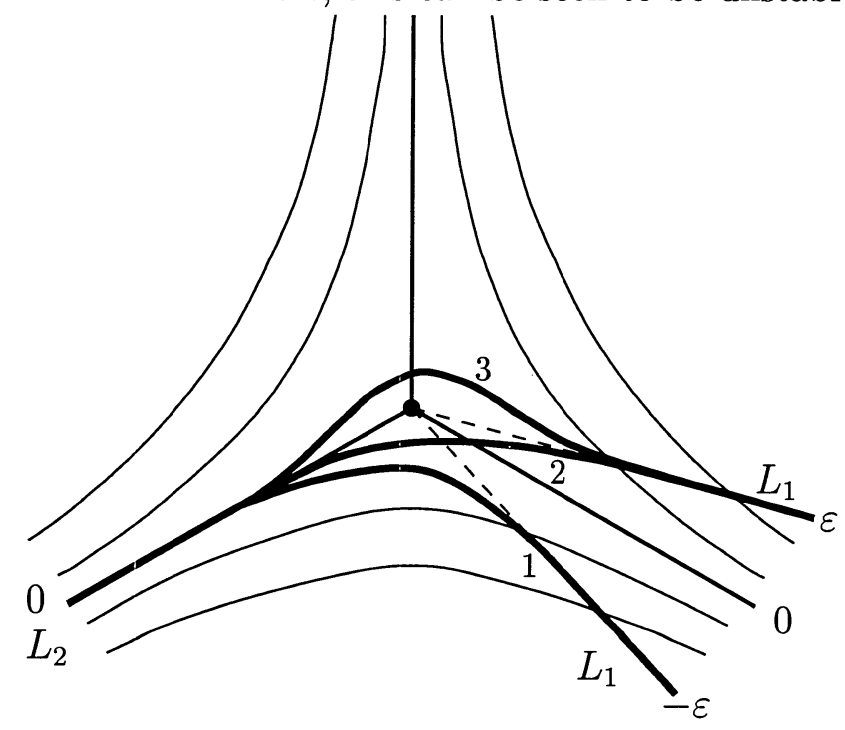

Figure 4: The two connect sums $L_{1} \# L_{2}$ and $L_{2} \#\left(L_{1}[-1]\right)$ in 3-dimensions

Things are not quite as simple as we have portrayed them if the initial curve has very large phase variation. It is quite possible for a curve corresponding to a stable Lagrangian, which is nonetheless very far from being a SLag, to pass close to a zero of $p$ without the large negative curvature away from the zero that the SLags exhibit. It can then flow into the zero, the Lagrangian being split into unions of Lagrangians of which it was a connect sum, despite their phases being such that they do not destabilise it. This limit is in the closure of the hamiltonian deformation orbit of the original Lagrangian, but does not contradict stability.

(A similar often-ignored subtlety occurs with stable bundles: when moduli of semistable sheaves are created by using GIT on part of a Quot scheme, the orbits of stable sheaves are not closed in Quot - the closures contain gradeds coming from any extension of sheaves forming the sheaf, stable or not. 
It is only in the locus of points of Quot representing semistable sheaves that the stable orbits are closed.)

The point about stable Lagrangians is that this can be avoided by choosing a hamiltonian deformation of the Lagrangian to have sufficiently small variation in phase $\theta$ (or sufficiently small volume) that it cannot be split into destabilising Lagrangians of different phase (or higher total volume); this we discuss now.

\section{The conjecture.}

It is now clear what our conjecture should be. Fix a (graded) Lagrangian submanifold $L$ of a Calabi-Yau $n$-fold $X$, and choose the phase of $\Omega$ such that the cohomological phase $\phi(L)=0$. Suppose first that the variation in $L$ 's phase function $\theta$ is sufficiently small in the sense that

$$
\left[\phi\left(L_{1}\right), \phi\left(L_{2}\right)\right] \nsubseteq\left(\inf _{L} \theta, \sup _{L} \theta\right),
$$

for all graded connect sums $L_{1} \# L_{2} \approx L$ (by this we mean either the pointwise connect sums of Section 3 or one of the relative connect sums discussed in [Th]). This condition (7.1) is preserved by the flow, by the maximum principle and equation (2.5), and so prohibits $L$ splitting up as a connect sum under the flow, as in the limit of flowing to such a splitting (7.1) would be violated.

We can also usefully consider volume instead of phase. If the Riemannian volume of our Lagrangian $L$ is less than the cohomological volume of any decomposition:

$$
\operatorname{vol}(L) \leq \int_{L_{1}} e^{-i \phi\left(L_{1}\right)} \Omega+\int_{L_{2}} e^{-i \phi\left(L_{2}\right)} \Omega,
$$

for all $L_{1}, L_{2}$ such that $L \approx L_{1} \# L_{2}$, then we again expect convergence of mean curvature flow to a SLag representative for $L$. This is also preserved under the flow, by (2.6), and so precludes the flow splitting $L$ into $L_{1} \cup L_{2}$, as this splitting would necessarily have higher volume. (As the referee pointed out, this is a global condition on $L$, whereas (7.1) appears to be a pointwise one. The global nature of (7.1) is that the points concerned are the extrema of $\theta$ over all of $L$.)

Conjecture 7.3. If $L$ satisfies either of the conditions (7.1) or (7.2) then mean curvature flow for $L$ exists for all time and converges to a special Lagrangian in its hamiltonian deformation class; the unique SLag conjectured in $[\mathrm{Th}]$. 
It is of course a consequence of this and the conjecture in [Th] that some hamiltonian deformation of $L$ satisfies (7.1) if and only if it is stable. The SLag should also be unique in its hamiltonian deformation class as in Theorem 4.3. If $L$ is stable but not close enough to being SLag that (7.1) fails, then mean curvature flow can become singular in finite time, (locally) splitting the Lagrangian in the reverse of a connect sum operation (i.e. with a vanishing cycle which is an $S^{n-1}$, or an $S^{n-r}$-bundle over an $(r-1)$-dimensional base in the relative connect sum case). We might then conjecture that the resulting pieces are smooth so we can begin the process again until we get a decomposition into different phase SLags. Typically, in the simplest case, we would get $L_{1} \cup L_{2}$ (with $\phi\left(L_{1}\right)<\phi\left(L_{2}\right)$ by stability) which is not a hamiltonian deformation of $L$ (though it is in the closure of such deformations).

If $L$ is unstable, we would again expect such finite time singularities and SLag splittings. But if $L$ 's phase variation, or volume, is sufficiently small, we can hope for convergence to the Jordan-Hölder decomposition of Section 5.3. That is, while the volume of $L$ must be larger than the cohomological volume of its Jordan-Hölder decomposition, if it is less than any other decomposition then it can only flow to the former. Again we expect the flow to become singular in finite time, the limit (locally) splitting $L$ into pieces for which we restart the flow. This splitting of the Lagrangian is a manifestation of the well known finite-time dumb-bell singularities in mean curvature flow.

\section{Proof for our example.}

Under our flow (6.9) in the Shapere-Vafa example the proofs of the evolution equations for the phase function $\theta(2.5)$ and volume (2.6) show that the equations are modified (as the metric is not quite Ricci-flat) to

$$
\begin{aligned}
\frac{d}{d t} \theta & =-\Delta \theta+\frac{\langle d \theta, d|\Omega|\rangle}{|\Omega|} \\
\frac{d}{d t}\left(|\Omega| \operatorname{vol}_{L}\right) & =-|d \theta|^{2}\left(|\Omega| \operatorname{vol}_{L}\right) .
\end{aligned}
$$

when $|\Omega|=|\Omega / \mathrm{vol}|$ is not $\equiv 1$. Therefore the maximum principle still holds for $\theta$, and the condition (7.1) is again preserved by the flow. Similarly if we measure volume with respect to $|\Omega| \operatorname{vol}_{L}$ then this is decreasing and (7.2) is preserved by the flow. We can now prove the appropriate version of our conjecture in this example. From the proof it will also be clear that the original conjecture could be proved in this case in the $O(n)$-invariant Ricci 
flat metric if we knew it, we would just not be able to be as explicit about the flow equations.

Theorem 7.6. Suppose that $\gamma$ is a curve in $\mathbb{C}$, with endpoints at zeros of $p$, and otherwise missing the zeros of $p$, such that its pointwise phase $\theta$ (6.4) satisfies (7.1) for all Lagrangians $L_{i}=\gamma_{i}^{n}, i=1,2$, fibred over curves $\gamma_{i}$ in the base, and also $S-I:=\sup _{\gamma} \theta-\inf _{\gamma} \theta<2 \pi / 3$. Then the flow (6.9) exists for all time and converges in $C^{\infty}$ to a smooth curve whose phase function (6.4) is constant.

We break the proof up into existence of the flow (best dealt with at the level of $\gamma^{n} \subset X^{n}$ ), controlling the angle variation (using $\gamma \subset \mathbb{C}$ ) to ensure no $180^{\circ}$ kinks appear in $\gamma$, and using this to show the flow exists for all time (for which we use the double cover $\gamma^{1} \subset X^{1}$ and $\theta$ on $\gamma^{n}$ ). We follow [An], in parts heavily modified to take care of the endpoints of $\gamma$. Finally we will show that the flow converges to a SLag.

Lemma 7.7. The flow (6.9) exists while the curvature of $\gamma^{1}$ is bounded.

Proof Firstly, short term existence of the flow, given any initial curve $\gamma \subset \mathbb{C}$ missing the zeros of $p$ except at its endpoints and such that $\gamma^{1}$ is $H^{2+\alpha}$ for some $\alpha>0$ (i.e. $\gamma^{1}$ has Hölder continuous curvature), is in fact most easily proved at the level of the $H^{2+\alpha}$ Lagrangian $\gamma^{n}$; see [An] for the method in 1 dimension (which easily generalises to $n$ dimensions), and [Ch] for a similar $n$-dimensional result. This is also done in ([Sm] Proposition 1.6) using results of Hamilton [H], for instance.

While the curvature of the curve $\gamma^{1} \subset X^{1}$ is bounded, so is the norm of the flow vector (the last term in (6.9) is always bounded, and the second term can be bounded by the curvature at an intermediate point by Taylor's theorem). So at any finite time $T$ the flow converges to a limit curve $\gamma_{T}^{1}$ pointwise. Parametrising the curves by their arclength on $X^{1}$, their first and second derivatives as maps to $X^{1}$ are therefore bounded, which by ArzelàAscoli implies that for a subsequence of $t$ we have convergence in $C^{1}$ to a $C^{1}$ curve with bounded (weak) curvature. By the uniqueness of the limit, then, $\gamma_{T}^{1} \subset X^{1}$ has bounded curvature.

Bounds on (the derivative of) the phase of $\gamma^{1}$ give corresponding bounds on (the derivative of) the phase of $\gamma^{n}$ (via (6.9) for $n$ and $n=1$ ). So the phase function $\theta$ of $\gamma^{n}$ is also $C^{0}$ convergent to the phase of $\gamma_{T}^{n}$, and satisfies the parabolic equation (7.4). Putting this into local coordinates and differentiating with respect to arclength $s$, we get a uniformly parabolic equation with bounded coefficients and a bounded solution $\theta_{s}$ on $t \in[0, T]$. 
By ([LSU] Section III Theorem 10.1), then, $\theta_{s}$ is in fact $\alpha$-Hölder continuous for some $\alpha>0$, and $\gamma_{T}^{1}$ is $H^{2+\alpha}$. By the existence of the flow for $H^{2+\alpha}$ initial conditions, then, the flow exists for some time $t>T$.

Lemma 7.8. $\limsup \left|s-s^{\prime}\right| \rightarrow 0\left|\theta\left(\gamma^{\prime}(s, t)\right)-\theta\left(\gamma^{\prime}\left(s^{\prime}, t\right)\right)\right|<\pi$ for all time $t$ for which the flow exists, where $s$ is arclength along $\gamma(., t)$, and $\theta\left(\gamma^{\prime}\right)$ is the argument of $\gamma^{\prime} \in \mathbb{C}$.

Proof Working outside a fixed neighbourhood of the zeros of $p$ at the endpoints of $\gamma$, this follows from the bounds on $\theta=\theta\left(\gamma^{\prime}\right)+(n / 2-1) \theta(p(\gamma))(6.4)$ coming from the $\sup \theta-\inf \theta<2 \pi / 3$ assumption (preserved under the flow by the maximum principle (7.4)), as the variation of $\theta(p(\gamma))$ can be made arbitrarily small with $\left|s-s^{\prime}\right|$. Since $\gamma$ must stay at a bounded distance from other zeros of $p$ by the condition (7.1) and the maximum principle for $\theta$ (7.4), we are left with proving the lemma in an arbitrarily small neighbourhood in $\mathbb{C}$ of the endpoints of $\gamma$.

Unfortunately, in this region, the bounds we want for $\theta\left(\gamma^{\prime}\right)$ do not follow from the bounds we have for $\theta$, and in fact come only from comparison with known solutions. Draw the SLags of phase $S, I$ emanating from a zero of $p$, i.e. the curves in $\mathbb{C}$ solving $\theta\left(\gamma^{\prime}\right)+(n / 2-1) \theta(p(\gamma))=S$ or $I$. (Since this is an ODE, there is no problem in finding solutions and extending them to either infinity or another zero of $p$; see [SV].) In the tangent space to the zero of $p$ this gives a cone of angle $(S-I) / n<2 \pi / 3 n$ which $\gamma$ lies inside and cannot cross either at $t=0$ or any later time in the flow. So in a sufficiently small neighbourhood of an endpoint of $\gamma$, we may bound $\theta(\gamma)$ inside a cone of angle less than $2 \pi / 3 n$, and also take $\theta(p(\gamma))$ to be within any given $\varepsilon$ of $\theta(\gamma)+C$ (since the zero of $p$ is nondegenerate; here $C$ is the phase of $\dot{p}$ at the zero of $p)$. Thus $\theta(p(\gamma))$ can be bounded inside a similar cone, so that (6.4) bounds the variation of $\theta\left(\gamma^{\prime}\right)$ by $2 \pi / 3+(n / 2-1) 2 \pi / 3 n<\pi$.

The bounds on $\theta\left(\gamma^{\prime}\right)$ imply that the curve does not spiral round its endpoints but moves away from them with nonzero derivative inside the above cone until it is outside the small neighbourhood employed above. So the remaining case to consider is if the curve can pass arbitrarily close to one of its own endpoints at some bounded-below arclength from its endpoints, i.e. if the cone of SLags above starting from a zero of $p$ passes either side of that same zero at some nonzero arclength. Then there would be a Slag fibred over a curve starting and ending at the same root of $p$, with our Lagrangian $\gamma^{n}$ the connect sum of this SLag and some other $\gamma_{1}^{n}$. But $\gamma^{n}$ cannot flow arbitrarily close to such a connect sum as its phase variation would approach at least the difference between the phase of the SLag and $\phi\left(\gamma_{1}^{n}\right)$, contradicting 
(7.1).

By Lemma 7.7 the flow exists for all time unless, as we suppose now, the curvature of $\gamma^{1}$ becomes unbounded in finite time. Then to get a contradiction we start by scaling as in [An]. Pick $s_{i}, t_{i}, i=1,2, \ldots$ such that $t_{i}$ tends to the blow up time and the curvature $\kappa_{i}$ of $\gamma^{1}\left(s_{i}, t_{i}\right)=y_{i}$ is maximal over the curvatures of $\gamma^{1}(s, t)$ for all $s$, and all $t \leq t_{i}$ (here we parameterise by arclength $s$ on $X^{1}$, centred at a zero $z$ of $p$, i.e. $\left.\gamma^{1}\right|_{s=0}=z$ lies over an endpoint of $\gamma$ ).

How we handle the blow up depends on whether it happens at the branch points of $\gamma^{1}$ (i.e. the endpoints of $\gamma$ ), by which we mean $\left|s_{i}\right|=O\left(\left|\kappa_{i}^{-1}\right|\right)$, or in the interior $\left|s_{i}\right| \gg\left|\kappa_{i}^{-1}\right|$, due to the different nature of (6.9) at the branch points. We first deal with the interior where the flow is a perturbation of mean curvature flow and so can be handled by [An]:

Lemma 7.9. Supposing that the curvature blows up as above, then $\left|s_{i} \kappa_{i}\right|$ is bounded.

Proof Firstly, if after passing to a subsequence of $i \in \mathbb{N}$, and centring $s$ about the other branch point of $\gamma^{1}$ if necessary, the blow up occurs at a finite distance $\left|s_{i}\right|>\varepsilon>0$ from either branch point of $\gamma^{1}$ then in this interior the flow (6.9) is a finite perturbation of mean curvature flow satisfying the conditions of [An], so a $180^{\circ}$ kink must appear in $\gamma^{1}$, contradicting Lemma 7.8. So we need only deal with the case of $s_{i} \rightarrow 0$ (by passing to a subsequence to concentrate around one of the two branch points, if necessary) while $r_{i}:=\left|s_{i} \kappa_{i}\right| \rightarrow \infty$.

Then we rescale as in $[\mathrm{An}]$;

$$
s \mapsto \kappa_{i} s, \quad g \mapsto \kappa_{i} g, \quad t \mapsto \kappa_{i}^{2}\left(t-t_{i}\right),
$$

where $g$ is the metric on $X^{1}$. This rescaled flow for $\gamma_{i}^{1}$ has the same form as $(6.9)$

$$
\dot{\gamma}_{i}^{1}=\operatorname{MCV}-(n-1) \mathbf{n}\left(\log |p|_{i}^{1 / 2}\right) \mathbf{n}+\frac{1}{2} \mathbf{n}\left(\log \left(|p|_{i}+|\dot{p}|_{i}^{2} / 4\right)\right) \mathbf{n},
$$

but with $|p|$ and $|\dot{p}|$ replaced by their pullbacks $|p|_{i},|\dot{p}|_{i}$ to the new Riemannian surface (here it is important that $\dot{p}$ is still computed in the old coordinates, then pulled back). Therefore their gradients are scaled by $\kappa_{i}^{-1}$. $\mathbf{n}$ denotes the unit normal to $\gamma^{1}$ in the new metric on $X^{1}$. The curvature gets scaled by $\kappa_{i}^{-1}$ and so has a maximum, over $t \leq 0$, of 1 at $y_{i}$ (at time 
$t=0)$. We want to show that the two perturbation terms on the right hand side of the above flow tend to zero as $i \rightarrow \infty$.

In the rescaled variables, work in a geodesic disc in $X^{1}$ of radius $r_{i} / 2$ $\left(r_{i}=\left|\kappa_{i} s_{i}\right| \rightarrow \infty\right.$ as $\left.i \rightarrow \infty\right)$ about $y_{i}$. As $s_{i} \rightarrow 0$, for $i$ sufficiently large this is within an arbitrarily small neighbourhood of $z$ in the original metric, in which $\gamma^{\prime}$ varies within an angle $<\pi$ cone as in the proof of Lemma 7.8, i.e. $\left(\gamma^{1}\right)^{\prime}$ varies within an angle $<\pi / 2$ cone on the double cover $X^{1}$. So arclength $s$ on $\gamma^{1}$ and radial distance $r$ in $X^{1}$ are equivalent metrics on $\gamma^{1}$ in this disc; $r_{s}:=\partial r / \partial s$ and $s_{r}=\partial s / \partial r$ are both bounded.

As $y_{i} \in \gamma_{i}^{1}$ is of arclength $s_{i} \geq r_{i}$ from $z$ (the zero of $p$ ) at $s=0$, we deduce that all points of our disc are of distance $\geq c r_{i} / 2$ from the zero of $p$ (for some constant $c>0$ fixed for all $i \gg 1$ ) in the new metric. Thus, for $i$ large enough, we have

$$
|p|_{i}^{1 / 2} \geq C \kappa_{i}^{-1}\left(c r_{i} / 2\right)
$$

where $C$ is a constant just less than the norm of the derivative of $p^{1 / 2}$ at the zero $z$ in the original metric on $X^{1}\left(p^{1 / 2}\right.$ pulls back to a well defined function on $X^{1}$ with a simple zero at $z$ ). We can therefore bound

$$
\left|\dot{\gamma}_{i}^{1}-\mathrm{MCV}\right| \leq(n-1) \frac{\kappa_{i}^{-1} \sup \left|d\left(p^{1 / 2}\right)\right|}{C \kappa_{i}^{-1}\left(c r_{i} / 2\right)}+\frac{1}{2} \kappa_{i}^{-1} \sup \left|d \log \left(|p|+|\dot{p}|^{2} / 4\right)\right|,
$$

where both sups are taken over small neighbourhoods of $z$ in the original metric on $X^{1}$. As $i \rightarrow \infty, \kappa_{i}, r_{i} \rightarrow \infty$, so the above bound tends to zero, while the radius of the disc we are working on $r_{i} / 2 \rightarrow \infty$. It follows that in the limit we get mean curvature flow of a curve inside an infinite flat disc $\mathbb{R}^{2}$; see ([An] Section 9) for how to pass to the limit to conclude that for this blow up to occur a $180^{\circ}$ kink must appear in the curve $\gamma^{1}$ (by which we mean the limsup in Lemma 7.8 is $\geq \pi$ ). But this contradicts Lemma 7.8.

(We do not repeat Angenent's argument here as we will give a slightly harder, $n$-dimensional, version of it around the endpoints of $\gamma$ in Lemma 7.11 below. The point is just that in the rescaling we can get rid of the last two terms of our flow to reduce to the results of $[\mathrm{An}]$.)

The remaining case we must dismiss is that of $\kappa_{i}$ blowing up at points $y_{i}=\gamma^{1}\left(s_{i}, t_{i}\right)$ with $\left|s_{i}\right|<A /\left|\kappa_{i}\right|$ for some fixed $A$. Here we must work harder than $[\mathrm{An}]$.

Lemma 7.11. The curvature of $\gamma^{1}$ does not blow up in finite time.

Proof By Lemma 7.8 we know that there is an $A$ such that $\left|s_{i}\right|<A /\left|\kappa_{i}\right|$. We rescale variables as in (7.10), and work on a length $\kappa_{i}^{1 / 2} \rightarrow \infty$ interval (in 
the new metric) on $\gamma^{1}$ centred $(s=0)$ at the zero $z$ of $p$. This is contained inside the ball of radius $\kappa_{i}^{-1 / 2} \rightarrow 0$ about $z$ in $X^{1}$ in the original metric, so for $i$ sufficiently large we can assume that $p(t)-C t$ is arbitrarily small in $C^{2}$ norm (here $t \in \mathbb{C}$ is the base parameter, not time, $C=\dot{p}(z)$, and the same is true of any $C^{r}$ norm; $r=2$ is the case of interest for us). We start by obtaining bounds on the polar angle of the curve and its tangent vector. We shall confuse functions on $\mathbb{C}$ with their pullbacks to $X^{1}$ (so writing things like $p\left(\gamma^{1}\right)$ etc.).

Taking $i$ sufficiently large that the metric on the radius $\kappa_{i}^{1 / 2}$ disc about $z$ in $X^{1}$ is sufficiently close to being flat, define geodesic polar coordinates on $X^{1}, r^{1}:=\left|\gamma^{1}\right|, \theta^{1}:=\theta\left(\gamma^{1}\right)$ (which is $\theta(\gamma) / 2$ to within a constant). Then we can assume that $\theta_{s}^{1}$ is arbitrarily $C^{1}$ close to

$$
\frac{1}{r} \sin \left(\theta\left(\gamma_{s}^{1}\right)-\theta^{1}\right)
$$

which is the exact formula for a flat metric and polar coordinates. This bounds $\left|r \theta_{s}^{1}\right|$. Since by construction the curvature of $\gamma^{1}$ is not more than one, i.e. $\left|\left(\theta\left(\gamma_{s}^{1}\right)\right)_{s}\right| \leq 1$, we can bound $\left|\theta\left(\gamma_{s}^{1}\right)\right| \leq s$.

Note that (7.12) in flat space gives us the differential equation

$$
f_{s}=\kappa_{i}-\frac{\sin f}{r}
$$

for $f=\theta\left(\gamma_{s}^{1}\right)-\theta^{1}$, with $f(0)=0$ and $\left|\kappa_{i}\right| \leq 1$. This implies that $|f(s)| \leq|s|$ (consider a point where the graph of $f$ crosses that of $\pm s$, where $\left|f_{s}\right| \geq 1$, for a contradiction), so for $i$ sufficiently large that our polar coordinates are sufficiently close to flat coordinates we can deduce a bound on $\left|\theta\left(\gamma_{s}^{1}\right)-\theta^{1}\right| / s$. Thus $\theta^{1} / s$ is also bounded, and $\theta^{1} / r$ by the uniform comparison bounds of $r$ and $s$ given by the cone argument in Lemma 7.8.

Instead of considering the equation (6.9) for $\gamma^{1}$, we analyse the equation (7.4) for $\theta$ on $\gamma^{n}$. After rescaling it becomes

$$
\dot{\theta}=\Delta \theta+\frac{\left\langle d \theta, d\left(|\Omega|_{i}\right)\right\rangle}{|\Omega|_{i}},
$$

where $|\Omega|_{i}$ is the pullback of $|\Omega|:=\mid \Omega /$ vol $\mid$ to $\gamma^{1}$ with its new metric. Note also that pulling functions up from $\gamma$ and taking their exterior derivative $d$ on either $\gamma^{1}$ or on $\gamma^{n}$ gives the same result via the obvious inclusion $\gamma^{1} \subset \gamma^{n}$ commuting with the projections to $\gamma$. Again we want to control this evolution equation as $i \rightarrow \infty$.

$|d \theta|=\left|\theta_{s}\right|=\left|\partial_{s}\left[\theta\left(\gamma_{s}^{1}\right) / 2+(n / 2-1) \theta\left(p\left(\gamma^{1}\right)\right)\right]\right|$, so this is bounded by the estimates above, for $i$ sufficiently large that $\theta\left(p\left(\gamma^{1}\right)\right)$ is $C^{1}$ close to $\theta^{1} / 2+$ const. 
in the disc in which we are working. So we can bound the last term in (7.13) by a constant times

$$
\kappa_{i}^{-1} \frac{\sup |d \Omega|}{\inf |\Omega|}
$$

where the sup and inf are taken in the original metric over a small neighbourhood of $(0, \ldots, 0, z) \in X^{n}$. This tends to zero as $i \rightarrow \infty$.

Computing the Laplacian on the space $\gamma^{n}$, with a radial coordinate $s$ and rotational symmetry about the origin $s=0$, makes (7.13)

$$
\theta_{t}=\theta_{s s}+(n-1) \frac{R_{s}^{i}}{R^{i}} \theta_{s}+O\left(\kappa_{i}^{-1}\right),
$$

where $R^{i}=R^{i}(s)$ is the radius of the sphere $S^{n-1}$ at $s$ in the new metric.

To compute $R^{i}$, we use our $\gamma^{1} \subset X^{1}$ arclength coordinate $s$, the radial coordinate $r$ on $X^{1}$, and a radial coordinate $\rho$ on $\mathbb{C}$. For $i$ sufficiently large, for $s \leq \kappa_{i}^{1 / 2}$ in the new metric, we can approximate $p$ linearly about $z$ and so assume that $R^{i}$ is as close as we like to $\kappa_{i} \sqrt{|\dot{p}(z)| \rho}$ in $C^{2}$. Therefore $R_{r}^{i}$ is approximated by

$$
R_{r}^{i}=\frac{R_{\rho}^{i}}{r_{\rho}}=\frac{R_{\rho}^{i}}{\sqrt{\kappa_{i}^{2}+\left(R_{\rho}^{i}\right)^{2}}}=\frac{1}{\sqrt{1+\frac{\kappa_{i}^{2}}{\left(R_{\rho}^{i}\right)^{2}}}} \approx \frac{1}{\sqrt{1+\frac{4 \rho}{|\dot{p}(z)|}}}
$$

which is bounded and tends to 1 in the interval $s \in\left[0, \kappa_{i}^{1 / 2}\right)$. Similarly $R_{r r}^{i}$ can be taken to be arbitrarily small on the same interval, for $i$ sufficiently large.

Since $r_{s}$ is bounded, this gives bounds on $R_{s}^{i}$, implying that, on passing to a subsequence if necessary, the functions $R^{i}(s)$ are convergent as $i \rightarrow \infty$ by the Arzelà-Ascoli theorem.

Note also that for all $i, R_{s}^{i}(0)=1$. But to preserve this in the limit, we must similarly bound $R_{s s}^{i}$. Differentiating $r_{s}^{2}+r^{2}\left(\theta_{s}^{1}\right)^{2}=1$ and $R_{s}^{i}=R_{r}^{i} r_{s}$ gives

$$
R_{s s}^{i}=R_{r r}^{i} r_{s}^{2}-R_{r}^{i}\left(r\left(\theta_{s}^{1}\right)^{2}+r^{2} \theta_{s}^{1} \theta_{s s}^{1} / r_{s}\right) .
$$

We have bounded $R_{r r}^{i}, r_{s}, r_{s}^{-1}, r \theta_{s}^{1}$ and $\theta_{s}^{1}$; all this leaves is the last term in (7.16).

We have approximated $\theta_{s}^{1}$ in $C^{1}$ by $\frac{1}{r} \sin \left(\theta\left(\gamma_{s}^{1}\right)-\theta^{1}\right)$; differentiating approximates $r^{2} \theta_{s}^{1} \theta_{s s}^{1} / r_{s}$ as closely as we like $($ as $i \rightarrow \infty)$ to

$$
\frac{r \theta_{s}^{1}}{r_{s}}\left(\kappa_{i}-\theta_{s}^{1}\right) \cos \left(\theta\left(\gamma_{s}^{1}\right)-\theta^{1}\right)-\theta_{s}^{1} \sin \left(\theta\left(\gamma_{s}^{1}\right)-\theta^{1}\right),
$$


which we have bounded already. In conclusion, after passing to a subsequence if necessary, $R^{i}$ is $C^{1}$ convergent to some $R$ with $R_{s}(0)=1$, and the phase function $\theta^{\infty}$ of the limit curve $\gamma_{\infty}^{1}$ (which exists by Arzelà-Ascoli since $\left|\gamma_{s}^{1}\right|=1$ and $\left|\gamma_{t}^{1}\right|$ is bounded by the bound on its curvature $\kappa$ ) satisfies the limit of (7.14):

$$
\theta_{t}^{\infty}=\theta_{s s}^{\infty}+(n-1) \frac{R_{s}}{R} \theta_{s}^{\infty} .
$$

But this is just the heat equation for $\theta$ on $\mathbb{R}^{n}$ with radial coordinate $s$ and the $O(n)$-invariant metric in which the radius of the $S^{n-1}$ fibre over $s$ is $R(s)$. By construction of the time rescaling (7.10) it exists for all time $t \leq 0$, and the solution $\theta^{\infty}$ is bounded. Also the metric is uniformly elliptic compared to the flat metric, as $R_{r}=1$ (7.15) and $r_{s}$ is bounded above and below away from zero. Therefore, by Moser's Harnack inequality [Mo], $\theta^{\infty}$ is in fact constant.

But for all $i, \max \theta_{s}=1$ by construction, and passing to a subsequence if necessary the point where the maximum is obtained is convergent. To show then that $\max \theta_{s}^{\infty}=1$, to get our contradiction, we need only know that $\theta_{s}$ is, say, uniformly (in i) Hölder continuous. This is again a consequence of ([LSU] Section III Theorem 10.1) as follows. By the boundedness of $\theta_{s}$ and the parabolic equation it satisfies (different for each $i$ ), $\theta_{s}$ is in fact $\alpha$-Hölder continuous for some $\alpha>0$, with $H^{\alpha}$ norm bounded by the bounds on the coefficients of the parabolic equations. But these are bounded uniformly in $i$, as a glance at (7.13) confirms: the correction term tends to zero, and the Laplacian term (and its derivative with respect to $s$ ) is controlled by $C^{2}$ bounds on the metric which we provided above by bounding $R^{i}(s), R_{s}^{i}$ and $R_{s s}^{i}$.

Finally we show that this infinite time flow converges using standard techniques (see for instance [C] for a harder result). Notice that the same scaling proof (7.11) that the curvature of $\gamma^{1}$ does not blow up in finite time shows the same for our now infinite time flow. So, using the $O(n)$ symmetry, the curvature of the metric on $\gamma^{n}$ stays uniformly bounded, and we have a $C^{1}$ bound on $\theta$. Therefore in the equation (7.4) for $\theta$ on $\gamma^{n}$, which we rewrite as

$$
\dot{\theta}=-\Delta^{\Omega} \theta:=|\Omega|^{-1} * d(|\Omega| * d \theta),
$$

the coefficients have at least uniform $C^{1}$ bounds; $\theta$ then acquires a uniform $C^{3}$ bound by parabolic Schauder estimates (see [LSU] III Theorem 12.1, for 
instance). Again by $O(n)$ symmetry we now get uniform $C^{2}$ bounds on $\gamma^{n}$ 's curvature. And so it goes on, giving $C^{\infty}$ bounds and allowing us to extract a subsequence of times for which the flow converges in $C^{\infty}$ to a Slag.

To see that the flow converges without having to pass to a subsequence we need only show convergence of $\theta$ in $L^{2}$; this way no other subsequence of the flow can converge to a different limit in $C^{\infty}$. In fact we use an $L^{2}$-norm weighted by $|\Omega|$, and compute using (7.18) and (7.5):

$\frac{d}{d t} \int_{\gamma^{n}}(\theta-\bar{\theta})^{2}|\Omega| \mathrm{vol}=\int_{\gamma^{n}}\left\{2(\theta-\bar{\theta})\left(-\Delta^{\Omega} \theta-\frac{d}{d t} \bar{\theta}\right)-(\theta-\bar{\theta})^{2}|d \theta|^{2}\right\}|\Omega|$ vol, where $\bar{\theta}=\int \theta|\Omega| \mathrm{vol} / \int|\Omega| \mathrm{vol}$ is constant on $\gamma^{n}$ (but not in time). This is then bounded above by

$$
-2 \int(\theta-\bar{\theta}) \Delta^{\Omega}(\theta-\bar{\theta})|\Omega| \text { vol. }
$$

It is easily checked that $\Delta^{\Omega}=d^{*^{\Omega}} d$, where $d^{*^{\Omega}}$ is the adjoint of $d$ with respect to the $L^{2}$-metric $\int_{\gamma^{n}}\langle\cdot, \cdot\rangle|\Omega|$ vol we are using. So its kernel is just the constants, and by the uniform $C^{\infty}$ bounds on the metric of $\gamma^{n}$ and $|\Omega|$ we can get a uniform lower bound $\lambda>0$ for its first nonzero eigenvalue. This then gives a bound $\int(\mu \Delta \mu)|\Omega|$ vol $\geq \lambda \int \mu^{2}|\Omega|$ vol for functions $\mu$ of integral zero. Setting $\mu=\theta-\bar{\theta}$ gives

$$
\frac{d}{d t} \int(\theta-\bar{\theta})^{2}|\Omega| \mathrm{vol} \leq-2 \lambda \int(\theta-\bar{\theta})^{2}|\Omega| \mathrm{vol},
$$

which therefore tends to zero as required. Theorem 7.6 is proved.

We end by noting that one can get cone-type bounds similar to those of Lemma 7.8 on the tangent direction $\theta\left(\gamma_{s}^{1}\right)$ even as a curve $\gamma$ representing an unstable Lagrangian $\gamma^{n}$ approaches and breaks across a zero of $p$. Suppose that the initial phase variation of $\gamma^{n}$ is, without loss of generality, in some $(-\delta, \delta)$. Draw the cone with boundary the SLags emanating from the zero of $p$ with phase $-\delta, \pi+\delta$ (the straight lines emanating from the zeros of $p$ drawn in Figures 2 and 4 display the $\delta=0$ cone for dimensions 2 and 3 respectively). Then in a sufficiently small neighbourhood of the zero, the variation in $\theta(p)$ can be taken to be less than $2 \pi / n+2 \delta+\varepsilon$ for any $\varepsilon>0$. Since we are free to make $\delta$ slightly smaller without violating the initial bounds on phase, we can ensure that there is a neighbourhood of the zero of $p$, and a cone with vertex at $p$ whose walls $\gamma$ cannot cross, such that for the part of $\gamma$ lying in this neighbourhood, the variation

$$
\sup \theta(p(\gamma))-\inf \theta(p(\gamma))<2 \pi / n+2 \delta .
$$


Comparing with (6.4) gives

$$
\sup \theta\left(\gamma^{\prime}\right)-\inf \theta\left(\gamma^{\prime}\right)<(n / 2-1)(2 \pi / n+2 \delta)+2 \delta=(1-2 / n) \pi+n \delta
$$

so that again no $180^{\circ}$ kinks can occur while this is less than $\pi$, i.e. for $\delta \leq 2 \pi / n^{2}$. So again the analysis should be tractable in this case (more general spiralling around a zero would make matters worse). However, we have not carried out the analysis necessary to show that at the moment $\gamma$ reaches the zero of $p$ it is sufficiently smooth that the two resulting curves it splits into give $C^{2}$ Lagrangians (whose flow we could restart).

Of course by just studying the simple examples above we cannot hope to know how bad the singularities are that arise in finite time in the general case. Also, as mentioned in [Th], we should perhaps restrict to those Lagrangians whose Floer cohomology is well defined [FO3]. This includes all homology spheres, however.

We should also point out the obvious fact that most of the evidence for our conjecture, other than perhaps the mirror symmetry and study of Joyce's examples in [Th], has been essentially one-dimensional (either for $T^{2}$ in [Th], or by symmetry reduction in the examples above). This is unrepresentative, essentially because the angles at which Lagrangians intersect (the $\alpha_{i}$ s of (3.3)) are all the same in this situation, and so are determined by the phase (their sum). So interesting phenomena, where degrees in Floer cohomology change (e.g. a Hom becomes an $\mathrm{Ext}^{i}$ on the mirror while the phase remains fixed) are largely lost due to them being controlled entirely by the phase.

\section{References.}

[An] S. Angenent, Parabolic equations for curves on surfaces. I. Curves with p-integrable curvature. Ann. of Math. (2) 132 (1990), 451-483.

[C] H. D. Cao, Deformation of Kähler metrics to Kähler-Einstein metrics on compact Kähler manifolds. Invent. Math. 81 (1985), 359-372.

[Ch] K.-C. Chang, Heat flow and boundary value problem for harmonic maps. Ann. Inst. H. Poincaré Anal. Non Linéaire 6 (1989), 363-395.

[FO3] K. Fukaya, Y.-G. Oh, H. Ohta, and K. Ono, Lagrangian intersection Floer theory - anomoly and obstruction, preprint.

[GH] P. Griffiths and J. Harris, Principles of algebraic geometry. Wiley, New York. 1978. 
[Gr] M. Grayson, Shortening embedded curves, Ann. of Math. 129 (1989), 71-111.

[H] R. S. Hamilton, Three manifolds with positive Ricci curvature, Jour. Diff. Geom. 23 (1986), 69-96.

[HaL] F. R. Harvey and H. B. Lawson, Calibrated Geometries, Acta Math. 148 (1982), 47-157.

[HuL] D. Huybrechts and M. Lehn, Geometry of moduli spaces of shaves. Aspects in Mathematics Vol. E31, Vieweg. 2000.

[KS] M. Khovanov and P. Seidel, Quivers, Floer cohomology, and braid group actions, J. Amer. Math. Soc. 15 (2002), 203-271.

[L] G. Lawlor, The angle criterion, Inv. Math. 95 (1989), 437-446.

[LSU] O. A. Ladyzhenskaya, V. A. Solonikov and N. N. Ural'ceva, Linear and quasilinear equations of parabolic type, Transl. Math. Monographs 23, AMS. 1968.

[Mi] J. Milnor, Lectures on the h-cobordism theorem. Princeton University Press. 1965.

[Mo] J. Moser, A Harnack inequality for parabolic differential equations, Comm. Pure Appl. Math. 17 (1964) 101-134.

[N] D. Nance, Sufficient conditions for a pair of planes to be area minizing, Math. Ann. 279 (1989) 161-164.

[Oh1] Y.-G. Oh, Mean curvature vector and symplectic topology of Lagrangian submanifolds in Einstein-Kähler manifolds, Math. Z. 216 (1994), 471-482.

[Oh2] Y.-G. Oh, Floer cohomology, spectral sequence and the Maslov class of Lagrangian embeddings, Internat. Math. Res. Notices 7 (1996), 305-346.

[P] M. Poźniak, Floer homology, Novikov rings and clean intersections, Ph.D. thesis, University of Warwick, 1994.

[SW] R. Schoen and J. Wolfson, Minimizing area among Lagrangian surfaces: the mapping problem. J. Differential Geom. 58 (2001), 1-86. 
[S1] P. Seidel, Lagrangian two-spheres can be symplectically knotted, Jour. Diff. Geom. 52 (1999), 145-171.

[S2] P. Seidel, Graded Lagrangian submanifolds, Bull. Soc. Math. France. 128 (2000), 103-146.

[S3] P. Seidel, Vanishing cycles and mutation, European Congress of Mathematics, Vol. II (Barcelona, 2000), 65-85, Progr. Math., 202, Birkhäuser, Basel, 2001.

[ST] P. Seidel and R. P. Thomas, Braid group actions on derived categories of sheaves, Duke Math. J. 108 (2001), 37-108.

[SV] A. Shapere and C. Vafa, BPS structure of Argyres-Douglas superconformal theories, preprint hep-th/9910182.

[Sm] K. Smoczyk, A canonical way to deform a Lagrangian submanifold, preprint dg-ga/9605005.

[SYZ] A. Strominger, S.-T. Yau and E. Zaslow, Mirror Symmetry is TDuality, Nucl. Phys. B479 (1996), 243-259.

[Th] R. P. Thomas, Moment maps, monodromy and mirror manifolds, Symplectic geometry and mirror symmetry (Seoul, 2000), 467-498, World Sci. Publishing, 2001.

[Wa] M. T. Wang, Deforming area-preserving diffeomorphisms of surfaces by mean curvature flow, Math. Res. Lett. 8 (2001), 651-661.

Department of Mathematics, Imperial College

Huxley Building, 180 QueEn's Gate, London, SW7 2BZ. UK

E-mail: richard.thomas@ic.ac.uk

Department of Mathematics, Harvard University

One Oxford Street, Cambridge MA 02138. USA

E-mail: yau@math.harvard.edu 